\title{
Family of equations of state based on lattice QCD: Impact on flow in ultrarelativistic heavy-ion collisions
}

\author{
M. Bluhm, ${ }^{1}$ B. Kämpfer, ${ }^{1,2}$ R. Schulze, ${ }^{2}$ D. Seipt, ${ }^{2}$ and U. Heinz ${ }^{3}$ \\ ${ }^{1}$ Forschungszentrum Dresden-Rossendorf, PF 510119, D-01314 Dresden, Germany \\ ${ }^{2}$ Institut für Theoretische Physik, TU Dresden, D-01062 Dresden, Germany \\ ${ }^{3}$ Department of Physics, The Ohio State University, Columbus, Ohio 43210, USA
}

(Received 6 May 2007; published 17 September 2007)

\begin{abstract}
We construct a family of equations of state within a quasiparticle model by relating pressure, energy density, baryon density, and susceptibilities adjusted to first-principles lattice QCD calculations. The relation between pressure and energy density from lattice QCD is surprisingly insensitive to details of the simulations. Effects from different lattice actions, quark masses, and lattice spacings used in the simulations show up mostly in the quark-hadron phase transition region, which we bridge over by a set of interpolations to a hadron resonance gas equation of state. Within our optimized quasiparticle model we then examine the equation of state along isentropic expansion trajectories at small net baryon densities, as relevant for experiments and hydrodynamic simulations at RHIC and LHC energies. We illustrate its impact on azimuthal flow anisotropies and on the transverse momentum spectra of various hadron species.
\end{abstract}

DOI: 10.1103/PhysRevC.76.034901

PACS number(s): 24.85.+p, 12.38.Mh, 25.75.-q

\section{INTRODUCTION}

In the past few years, much evidence has been accumulated for the applicability of hydrodynamics in describing the expansion stage of strongly interacting matter created in relativistic heavy-ion collisions [1-7]. Hydrodynamics describes the collective flow of bulk matter from an initial state just after reaching thermalization up to the kinetic freeze-out stage. The heart of hydrodynamics is the equation of state (EoS), which relates thermodynamically the pressure $p$ of the medium to its energy density $e$ and net baryon density $n_{B}$ (or, equivalently, to its temperature $T$ and baryon chemical potential $\mu_{B}$ ). Specifically, the parameter controlling the acceleration of the fluid, that is, the buildup of collective flow, by pressure gradients in the system is the speed of sound, given by $c_{s}^{2}=\frac{\partial p}{\partial e}$.

Although most existing hydrodynamic simulations have used a realistic hadron resonance gas EoS below the deconfinement transition (either with full [1,4,5] or partial [2,8-11] chemical equilibrium among the hadron species), they have usually relied on simple analytical models for the EoS of the quark-gluon plasma (QGP) above the transition, based on the assumption of weak coupling among the deconfined quarks and gluons. This assumption is, however, inconsistent with the phenomenological success of hydrodynamics, which requires rapid thermalization of the QGP [12] and therefore strong interactions among its constituents [13-16]. Indeed, lattice QCD calculations of the QGP pressure and energy density show that they deviate from the Stefan-Boltzmann limit for an ideal gas of noninteracting quarks and gluons even at temperatures $T>3 T_{c}$ (with $T_{c}$ as pseudo-critical temperature), by about 15-20\% [17-19]. Miraculously, however, the deviations are of similar magnitude in both $p$ and $e$ such that, for $T \gtrsim 2 T_{c}$, the squared speed of sound, $c_{s}^{2}=\frac{\partial p}{\partial e} \approx \frac{1}{3}$ [19], just as expected for a noninteracting gas of massless partons. In spite of the evidence for strong interactions among the quarks and gluons in the QGP seen in both $p(T)$ and $e(T)$, the stiffness and accelerating power of the lattice QCD equation of state is thus indistinguishable from that of an ideal parton gas (at least for temperatures $T \gtrsim 2 T_{c}$ ), such as the one used above $T_{c}$ in most hydrodynamical simulations.

However, at $T<2 T_{c}$ the speed of sound extracted from lattice QCD drops below the ideal gas value $c_{s}=1 / \sqrt{3}$, reaching a value that is about a factor of 3 smaller near $T_{c}$ [19]. This leads to a significant softening of the QGP EoS relative to that of an ideal massless gas exactly in the temperature region $T_{c}<T<2 T_{c}$ explored during the early stages of $\mathrm{Au}+\mathrm{Au}$ collisions at RHIC $[1,2,4,5,8]$. To explore the sensitivity of the flow pattern seen in the RHIC data to such details of the EoS near the quark-hadron phase transition, the hydrodynamic evolution codes must be supplied with an EoS that faithfully reproduces the lattice $\mathrm{QCD}$ results above $T_{c}$. To construct such an EoS and to test its influence on the collective flow generated in RHIC and LHC collisions are the main goals of this paper.

Our approach is based on the quasiparticle model [20-29], which expresses the thermodynamic quantities as standard phase-space integrals over thermal distribution functions for quasiparticles with medium-dependent properties. In the present paper we follow the philosophy [20-28] that the interaction effects in the QGP can be absorbed into the quasiparticle masses and a vacuum energy, all of which depend on the temperature and baryon chemical potential. This is known to produce good fits to the lattice QCD data both at vanishing [20-23] and nonvanishing [25-27] baryon chemical potential. However, because this approach uses on-shell spectral functions for the quasiparticles, it implicitly assumes zero residual interactions (i.e., infinite mean free paths) for them, which is inconsistent with the low viscosity and almost ideal fluid dynamical behavior of the QGP observed at RHIC. Peshier and Cassing [29] have shown that it is possible to generalize the quasiparticle description to include a finite (even large) collisional width in the spectral functions, without significantly affecting the quality of the model fit to the lattice QCD data for 
the EoS at $\mu_{B}=0$. Since hydrodynamics only cares about the EoS, but not about its microscopic interpretation, we here opt for the simpler, but equally successful approach using on-shell quasiparticles to fit the lattice QCD EoS.

The quasiparticle EoS for the QGP above $T_{c}$ does not automatically match smoothly with the hadron resonance gas EoS below $T_{c}$. Although the gap between the two branches of the EoS is much smaller here than for the previously used models, which assume noninteracting quarks and gluons above $T_{c}$ [1-5,8-11], a certain degree of ambiguity remains in the interpolation process. We explore a set of different interpolation prescriptions, yielding a family of equations of state that exhibit slight differences in the phase transition region, and study their dynamical consequences.

Our paper is organized as follows: In Sec. II we show that our quasiparticle model provides an efficient and accurate parametrization of lattice QCD results for $N_{f}=2$ flavors both at $\mu_{B}=0$ and $\mu_{B} \neq 0$. We also extract the isentropic expansion trajectories followed by fully equilibrated systems. In that section, the quasiparticle parametrization is continued below $T_{c}$, down to temperatures of about $0.75 T_{c}$ where the lattice QCD data end. In Sec. III we proceed to the physically relevant case of $N_{f}=2+1$ flavors and furthermore match the quasiparticle EoS above $T_{c}$ to a hadron resonance gas EoS below $T_{c}$. Variations in the matching procedure lead to a family of equations of state with slightly different properties near $T_{c}$. The transition to a realistic hadron resonance gas picture below $T_{c}$ means that these EoS can now be used down to much lower temperatures to make explicit contact with the experimentally observed final-state hadrons after decoupling from the expanding fluid. In Sec. IV we use this family of equations of state for hydrodynamic calculations of the differential elliptic flow $v_{2}\left(p_{T}\right)$ for several hadronic species in $\mathrm{Au}+\mathrm{Au}$ collisions at the top RHIC energy and compare the results with experimental data. We find some sensitivity to the details of the interpolation scheme near $T_{c}$, as long as an EoS is used that agrees with the lattice QCD data for energy densities $e>4 \mathrm{GeV} / \mathrm{fm}^{3}$. We conclude that section with a few predictions for $\mathrm{Pb}+\mathrm{Pb}$ collisions at the LHC. A short summary is presented in Sec. V.

\section{QUASIPARTICLE DESCRIPTION OF THE EQUATION OF STATE FROM LATTICE QCD FOR $N_{f}=2$}

\section{A. The quasiparticle model}

Over the years, several versions of quasiparticle models have been developed to describe lattice QCD data for the QCD equation of state [20-27,29]. They differ in the choice and number of parameters and in the details of the underlying microscopic picture but generally yield fits to the lattice QCD data that are of similar quality. In this section we quickly review the essentials of the model described in Ref. [20] that will be used here.

In our quasiparticle approach the thermodynamic pressure is written as a sum of contributions associated with medium modified light quarks $q$, strange quarks $s$, and gluons $g$ [20]:

$$
p\left(T,\left\{\mu_{a}\right\}\right)=\sum_{a=q, s, g} p_{a}-B\left(T,\left\{\mu_{a}\right\}\right),
$$

with partial pressures

$$
p_{a}=\frac{d_{a}}{6 \pi^{2}} \int_{0}^{\infty} d k \frac{k^{4}}{\omega_{a}}\left(f_{a}^{+}+f_{a}^{-}\right) .
$$

Here $f_{a}^{ \pm}=\left\{\exp \left(\left[\left(\omega_{a} \mp \mu_{a}\right) / T\right]+S_{a}\right\}^{-1}\right.$ are thermal equilibrium distributions for particles and antiparticles, with $S_{q, s}=1$ for fermions and $S_{g}=-1$ for bosons; $d_{a}$ represents the spin-color degeneracy factors, with $d_{q}=2 N_{q} N_{c}=12$ for the $N_{q}=2$ light quasiquarks, $d_{s}=2 N_{c}=6$ for the strange quasiquarks, and $d_{g}=N_{c}^{2}-1=8$ for the right-handed transversal quasigluons (with the left-handed ones counted as their antiparticles).

The mean-field interaction term $B\left(T,\left\{\mu_{a}\right\}\right)$ in Eq. (1), assuming all $T$ and $\left\{\mu_{a}\right\}$ dependence being incorporated in the self-energies $\Pi_{a}$ (see discussion in the following), is determined by thermodynamic self-consistency and stationarity of the thermodynamic potential under functional variation of the self-energies, $\delta p / \delta \Pi_{a}=0$ [30]. As a consequence, $B\left(T,\left\{\mu_{a}\right\}\right)$ is evaluated in terms of an appropriate line integral in the $T-\mu$ plane, with integration constant $B\left(T_{c}\right)$ adjusted to the lattice results [20].

Since the pressure integral in Eq. (2) is dominated by thermal momenta of order $k \sim T$, weak coupling perturbation theory suggests $[31,32]$ that the dominant propagating modes are transversal plasmons with gluon quantum numbers $(g)$ and quark-like excitations, whereas longitudinal plasmons are exponentially suppressed. Our model assumes that this remains true near $T_{c}$, where perturbation theory is not expected to be valid.

We are interested in the application of this EoS to heavy-ion collisions where strangeness is conserved at its initial zero value, owing to the very short available time. This strangeness neutrality constraint allows one to set $\mu_{s}=0$. The isospin chemical potential $\mu_{I}=\left(\mu_{u}-\mu_{d}\right) / 2$ is fixed by the net electric charge density of the medium; we assume zero net charge of the fireball matter created near midrapidity at RHIC as well as equal masses for the $u$ and $d$ quasiquarks such that $\mu_{I}=0$ and we have only one independent chemical potential $\mu_{u}=\mu_{d} \equiv \mu_{q}=\mu_{B} / 3$, where $\mu_{B}$ is the baryon number chemical potential.

The quasiparticles are assumed to propagate on-shell, that is, with real energies $\omega_{a}$ given by dispersion relations of the type $\omega_{a}=\sqrt{k^{2}+m_{a}^{2}\left(T, \mu_{q}\right)}$, known to hold for weakly interacting quarks and gluons with thermal momenta $k \sim T$. Again the model assumes that this structure holds true also near $T_{c}$, where perturbation theory presumably breaks down. To directly compare our quasiparticle model (QPM) with lattice QCD results, we include nonzero bare quark masses $m_{a 0}$ and adjust them to the values used in the lattice simulations through $m_{a}^{2}=m_{a 0}^{2}+\Pi_{a}$ [33]. For gluonic modes we use $m_{g 0}=0$. For $\Pi_{a}$ we employ an ansatz inspired by the asymptotic form of the gauge-independent hard thermal/dense loop (HTL/HDL) self-energies, which depend on $T, \mu_{q}, m_{a 0}$, and the running 
coupling $g^{2}$ as follows $[31,33]$ :

$$
\begin{aligned}
& \Pi_{g}=\left(\left[3+\frac{N_{f}}{2}\right] T^{2}+\frac{3}{2 \pi^{2}} \sum_{f} \mu_{f}^{2}\right) \frac{g^{2}}{6}, \\
& \Pi_{q}=2 m_{q 0} \sqrt{\frac{g^{2}}{6}\left(T^{2}+\frac{\mu_{q}^{2}}{\pi^{2}}\right)}+\frac{g^{2}}{3}\left(T^{2}+\frac{\mu_{q}^{2}}{\pi^{2}}\right), \\
& \Pi_{s}=2 m_{s 0} \sqrt{\frac{g^{2}}{6} T^{2}}+\frac{g^{2}}{3} T^{2} .
\end{aligned}
$$

The $m_{a}$ in the dispersion relations thus denote effective quasiparticle masses resulting from the dynamically generated self-energies $\Pi_{a}$.

All other thermodynamic quantities follow straightforwardly from the stationarity condition and standard thermodynamic relations. For example, the entropy density reads

$$
\begin{aligned}
s= & \sum_{a=q, s, g} s_{a}, \quad s_{a}=\frac{d_{a}}{2 \pi^{2}} \int_{0}^{\infty} k^{2} d k\left[\frac{\left(\frac{4}{3} k^{2}+m_{a}^{2}\right)}{\omega_{a} T}\left(f_{a}^{+}+f_{a}^{-}\right)\right. \\
& \left.-\frac{\mu_{a}}{T}\left(f_{a}^{+}-f_{a}^{-}\right)\right],
\end{aligned}
$$

and the net quark number density $n_{q}=3 n_{B}$ is given through

$$
n_{q}=\frac{d_{q}}{2 \pi^{2}} \int_{0}^{\infty} k^{2} d k\left(f_{q}^{+}-f_{q}^{-}\right) .
$$

Although the form of our ansatz for the quasiparticle masses (i.e., the specific interplay among the parameters $m_{a 0}, T$, and $\mu_{q}$ ) is inspired by perturbation theory, our model becomes nonperturbative by replacing the perturbative expression for the running coupling $g^{2}$ in Eqs. (3)-(5) by an effective coupling $G^{2}$ whose dependence on $T$ and $\mu_{q}$ is parametrized and fitted to the nonperturbative $\left(T, \mu_{q}\right)$ dependence of the thermodynamic functions from lattice QCD. The $\left(T, \mu_{q}\right)$ dependence of $G^{2}$ is constrained by Maxwell's relation for $p$, which takes the form of a quasilinear partial differential equation

$$
a_{\mu_{q}} \frac{\partial G^{2}}{\partial \mu_{q}}+a_{T} \frac{\partial G^{2}}{\partial T}=b ;
$$

here $a_{\mu_{q}}, a_{T}$, and $b$ depend on $T, \mu_{q}$, and $G^{2}$ (see Refs. [20,34] for details). This flow equation is solved by the method of characteristics, starting from initial conditions on a Cauchy surface in the $T-\mu_{q}$ plane. One possibility is to parametrize $G^{2}$ at $\mu_{q}=0$ such that lattice QCD results for vanishing quark chemical potential are reproduced, and to use the flow equation for extrapolation to nonzero $\mu_{q}$. As a convenient parametrization of $G^{2}\left(T, \mu_{q}=0\right)$ we find [35]

$$
G^{2}\left(T, \mu_{q}=0\right)=\left\{\begin{array}{l}
G_{2 \text { loop }}^{2}(T), \quad T \geqslant T_{c}, \\
G_{2 \text { loop }}^{2}\left(T_{c}\right)+b\left(1-\frac{T}{T_{c}}\right), T<T_{c} .
\end{array}\right.
$$

Here, to recover perturbation theory in the high-temperature limit, $G_{2-\text { loop }}^{2}$ is taken to have the same form as the perturbative running coupling at two-loop order:

$$
G_{2 \text { loop }}^{2}(T)=\frac{16 \pi^{2}}{\beta_{0} \log \xi^{2}}\left[1-\frac{2 \beta_{1}}{\beta_{0}^{2}} \frac{\log \left(\log \xi^{2}\right)}{\log \xi^{2}}\right],
$$

with $\beta_{0}=\frac{1}{3}\left(11 N_{c}-2 N_{f}\right)$ and $\beta_{1}=\frac{1}{6}\left(34 N_{c}^{2}-13 N_{f} N_{c}+\right.$ $\left.3 N_{f} / N_{c}\right)$. The scale $\xi$ is parametrized phenomenologically as $\xi=\lambda\left(T-T_{s}\right) / T_{c}$, with a scale parameter $\lambda$ and a temperature shift $T_{s}$ that regulates the infrared divergence of the running coupling by shifting it somewhat below the critical temperature $T_{c}$. Below the phase transition, we postulate a continuous linear behavior of the effective coupling. The parametrization (9) and (10) turns out to be flexible enough to describe the lattice QCD results accurately down to about $T \approx 0.75 T_{c}$. In contrast, using a pure one-loop or two-loop perturbative coupling together with a more complete description of the plasmon term and Landau damping restricts the quasiparticle approach to $T>2 T_{c}$ [36]. (Similar quality fits can be achieved in that approach, without giving up its more accurate form of the HTL/HDL self-energies, by adopting a similar nonperturbative modification of the running coupling as adopted here [23].)

It is worth pointing out that the discontinuity in the temperature derivative of the running coupling constant (9) at $T_{c}$ has no impact on the EoS because it is absorbed into the function $B\left(T, \mu_{q}\right)$ in Eq. (1) through the conditions of stationarity and thermodynamic consistency discussed below Eq. (2). The apparent singularity of Eq. (10) at $T=T_{s} \pm T_{c} / \lambda<T_{c}$ is never accessed because in Eq. (9) the singular function (10) is replaced by a smooth linear temperature dependence below $T_{c}$. Furthermore, we will later match the quasiparticle model parametrization above $T_{c}$ to a phenomenological hadron resonance gas EoS below $T_{c}$, thereby interpolating smoothly over these (apparent) singularities.

The model described in this section was successfully applied to QCD lattice data in the pure gauge sector in Ref. [20] and to first lattice QCD calculations at $\mu_{q} \neq 0$ in Ref. [37]. In the following section we test it on recent lattice QCD data for $N_{f}=2$ dynamical quark flavors at zero and nonzero $\mu_{q}$, and in the next section we consider the realistic case of $N_{f}=2+1$ flavors with the aim of providing an EoS suitable for hydrodynamic simulations of heavy-ion collisions.

\section{B. Thermodynamics of $N_{f}=2$ quark flavors}

We begin with the case of $N_{f}=2$ dynamical quark flavors at zero quark chemical potential and confront the QPM with lattice QCD results obtained by the Bielefeld-Swansea collaboration [17]. These simulations were performed with temperature-dependent bare quark masses $m_{a 0}(T)=x_{a} T$, where $x_{g}=0$ and $x_{q}=0.4$ [17]. For $N_{f}=2$ light quark flavors we can set $d_{s}=0$ in the QPM expressions. Figure 1 shows the lattice QCD data for the scaled pressure $p(T) / T^{4}$ together with the QPM fit; the fit parameters given in the caption were obtained by the procedure described in Ref. [38]. The raw lattice data were extrapolated to the continuum by multiplying the pressure in the region $T \geqslant T_{c}$ by a constant factor $d=1.1$, following an estimate given in Refs. [17,40], where a range of $10-20 \%$ is advocated because of finite size and cutoff effects. (Note that this estimated correction factor does not necessarily have to be independent of $T$, as assumed here.)

Having demonstrated the ability of the QPM to successfully reproduce lattice EoS data along the $\mu_{q}=0$ axis, we can now 


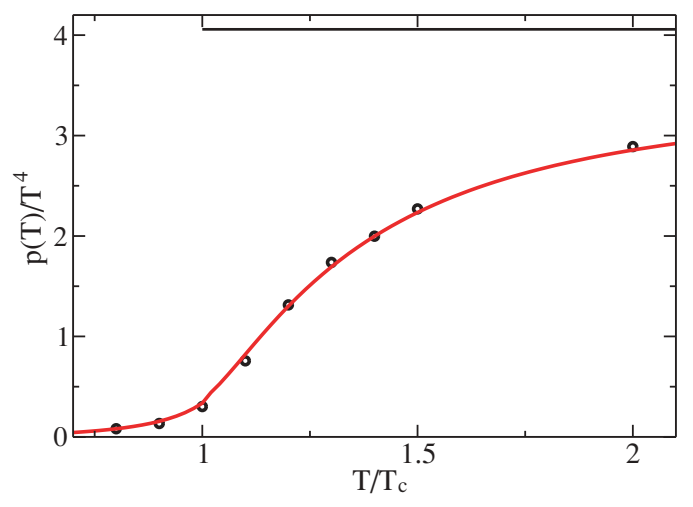

FIG. 1. (Color online) Comparison of the QPM with lattice QCD results (symbols) for the scaled pressure $p / T^{4}$ as a function of $T / T_{c}$ for $N_{f}=2$ and $\mu_{q}=0$. The raw lattice QCD data from Ref. [17] have been continuum extrapolated as described in the text. The QPM parameters are $\lambda=4.4, T_{s}=0.67 T_{c}, b=344.4$, and $B\left(T_{c}\right)=0.31 T_{c}^{4}$, with $T_{c}=175 \mathrm{MeV}$ as suggested in Ref. [39]. The horizontal line indicates the Stefan-Boltzmann value $p_{\mathrm{SB}} / T^{4}=\bar{c}_{0}=$ $\left(32+21 N_{f}\right) \pi^{2} / 180$ for $N_{f}=2$.

exploit recent progress in lattice QCD with small nonvanishing chemical potential to test its ability to correctly predict the thermodynamic functions at nonzero $\mu_{q}$. In Ref. [41] finite- $\mu_{q}$ effects were evaluated by expanding the pressure into a Taylor series in powers of $\left(\mu_{q} / T\right)$ around $\mu_{q}=0$,

$$
p\left(T, \mu_{q}\right)=T^{4} \sum_{n=0,2,4, \ldots}^{\infty} c_{n}(T)\left(\frac{\mu_{q}}{T}\right)^{n},
$$

where $c_{0}(T)=p\left(T, \mu_{q}=0\right) / T^{4}$ is the scaled pressure at vanishing quark chemical potential. The coefficients $c_{2}(T), c_{4}(T)$, and $c_{6}(T)$ were extracted from the lattice by numerically evaluating appropriate $\mu_{q}$ derivatives of the logarithm of the partition function $\ln Z=p V / T$ [41], that is,

$$
c_{n}(T)=\left.\frac{1}{n !} \frac{\partial^{n}\left(p / T^{4}\right)}{\partial\left(\mu_{q} / T\right)^{n}}\right|_{\mu_{q}=0} .
$$

These yield a truncated result for $p\left(T, \mu_{q}\right)$.

Note that computing the coefficients $c_{n}, n \geqslant 2$, from these expressions is easier on the lattice than determining the pressure at $\mu_{B}=0, c_{0}(T)$, because the latter requires an integration over $T$ and a separate lattice simulation at $T=0$. For this reason Ref. [41] has no results for $c_{0}(T)$. Since the simulations in Ref. [41] were done with different parameters than those analyzed in Fig. 1 [17], it is not immediately clear that the QPM parameters fitted to the results of Ref. [17] can also be used to describe the simulations reported in Ref. [41]. When analyzing the lattice data of Ref. [41] we therefore refit the QPM parameters to the lattice results for $c_{2}(T)$ (see dashed line and squares in Fig. 4) and then assess the quality of the model fit by its ability to also reproduce $c_{4}(T)$ and $c_{6}(T)$ extracted from the same set of simulations, as well as other thermodynamic quantities calculated from these coefficients through Taylor expansions of the type (11). The QPM parameters obtained by fitting $c_{2}(T)$ from Ref. [41] are [35] $\lambda=12.0, T_{s}=0.87 T_{c}$, and $b=426.05$ (again using $\left.T_{c}=175 \mathrm{MeV}\right)[42]$.
Evaluation of the derivatives in Eq. (12) within the QPM is straightforward; for explicit analytical expressions for $c_{2,4,6}(T)$ we refer the reader to Eqs. (6)-(8) in the second paper of Ref. [35]. That paper also shows that the quasiparticle model gives an excellent fit to $c_{2}(T)$ from Ref. [41], and that with the same set of parameters the QPM expressions for $c_{4}(T)$ and $c_{6}(T)$ yield impressive agreement with the lattice data [41], too. In particular, several pronounced structures seen in $c_{4}(T)$ and $c_{6}(T)$ are quantitatively reproduced owing to the change of curvature at $T=T_{c}$ of the effective coupling $G^{2}(T)$ in Eq. (9), which, as already pointed out in Ref. [35], introduces phasetransition-like signatures at $T_{c}$. This constitutes a stringent test of the efficiency of our QPM parametrization.

We here use these first three expansion coefficients $c_{2,4,6}(T)$ to write down truncated expansions for the net baryon density $n_{B}=\partial p / \partial \mu_{B}$ and the corresponding baryon number susceptibility $\chi_{B}=\partial n_{B} / \partial \mu_{B}$, which is a measure of fluctuations in $n_{B}$ :

$$
\begin{aligned}
\frac{n_{B}\left(T, \mu_{B}\right)}{T^{3}} \approx & \frac{2}{3} c_{2}(T)\left(\frac{\mu_{B}}{3 T}\right)+\frac{4}{3} c_{4}(T)\left(\frac{\mu_{B}}{3 T}\right)^{3} \\
& +2 c_{6}(T)\left(\frac{\mu_{B}}{3 T}\right)^{5}, \\
\frac{\chi_{B}\left(T, \mu_{B}\right)}{T^{2}} \approx & \frac{2}{9} c_{2}(T)+\frac{4}{3} c_{4}(T)\left(\frac{\mu_{B}}{3 T}\right)^{2} \\
& +\frac{10}{3} c_{6}(T)\left(\frac{\mu_{B}}{3 T}\right)^{4} .
\end{aligned}
$$

In Fig. 2, the truncated QPM results for $n_{B} / T^{3}$ and $\chi_{B} / T^{2}$ are compared for various values of $\mu_{B} / T_{c}$ with lattice QCD results that were obtained from Eqs. (13) and (14) with the coefficients $c_{2,4,6}(T)$ from Ref. [41]. We find good agreement with the lattice results; even below $T_{c}$, where our QPM parametrization is not well justified and should be replaced by a realistic hadron resonance gas (see Sec. III), the deviations are small but increase with increasing $\mu_{B} / T_{c}$. All in all, the QPM model appears to provide an efficient and economic parametrization of the lattice data down to $T \sim 0.75 T_{c}$.

Within the QPM we can assess the truncation error made in Eqs. (13) by comparing this expression with the exact result [Eq. (7)] (dashed lines in the upper panel of Fig. 2). The authors of Ref. [41] estimated the error induced in Eq. (11) by keeping only terms up to $n=4$ to remain $\leqslant 10 \%$ for $\mu_{B} / T \leqslant 3$. Here we keep the terms $\sim\left(\mu_{B} / T\right)^{6}$ and, as the upper panel of Fig. 2 shows, the resulting truncated expressions for the baryon density $n_{B}$ agree with the exact results within the line width as long as $\mu_{B} / T_{c} \leqslant 1.8$. For $\mu_{B} / T_{c}=2.4$ we see significant deviations between the truncated and exact expressions near $T=T_{c}$, which, however, can be traced back to an artificial mechanical instability $\partial p / \partial n_{B} \leqslant 0$ induced by the truncation. Similar truncation effects near $T=T_{c}$ are stronger and more visible in the susceptibility $\chi_{B}$ (lower panel of Fig. 2). In both cases the full QPM expression is free of this artifact and provides a thermodynamically consistent description.

We next compare the Taylor series expansion coefficients of the energy and entropy densities given in Ref. [39] with our 

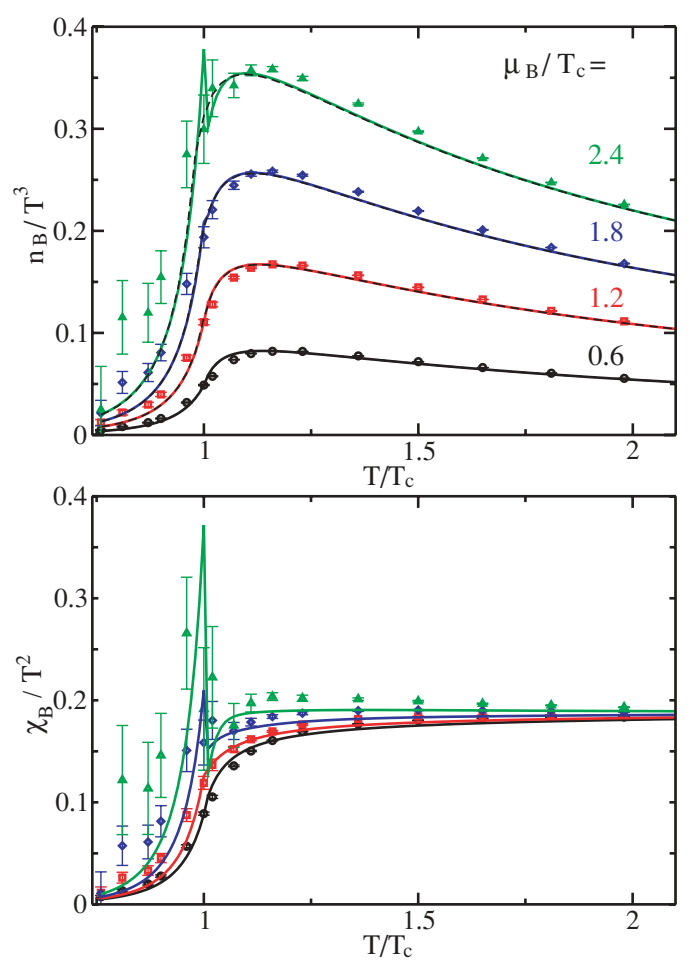

FIG. 2. (Color online) Scaled baryon density $n_{B} / T^{3}$ (upper panel) and baryon number susceptibility $\chi_{B} / T^{2}$ (lower panel) as a function of $T / T_{c}$, for $\mu_{B} / T_{c}=2.4,1.8,1.2$, and 0.6 (from top to bottom). QPM results from the truncated expansions (13) and (14) (solid lines) are compared with lattice QCD data (symbols) from ref. [41] for $N_{f}=2$. Dashed lines in the upper panel represent the full QPM result (7) for $n_{B}=n_{q} / 3$. The QPM parameters are $\lambda=12.0, T_{s}=0.87 T_{c}$, and $b=426.05$, for $T_{c}=175 \mathrm{MeV}$.

model. We have the following decompositions [39]:

$$
\begin{aligned}
& e=3 p+T^{4} \sum_{n=0}^{\infty} c_{n}^{\prime}(T)\left(\frac{\mu_{q}}{T}\right)^{n}, \\
& s=s\left(T, \mu_{q}=0\right)+T^{3} \sum_{n=2}^{\infty}\left[\left((4-n) c_{n}(T)+c_{n}^{\prime}(T)\right]\left(\frac{\mu_{q}}{T}\right)^{n},\right.
\end{aligned}
$$

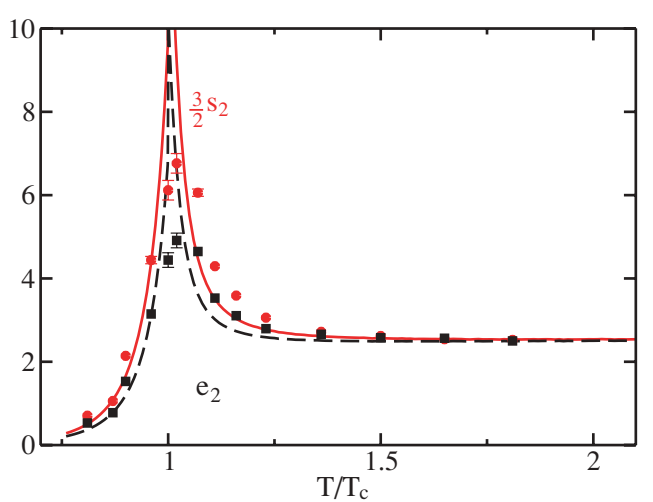

with $p$ from Eq. (11), $c_{n}^{\prime}(T)=T d c_{n}(T) / d T$, and

$$
s\left(T, \mu_{q}=0\right)=T^{3}\left[4 c_{0}(T)+c_{0}^{\prime}(T)\right] .
$$

Because these expressions contain both $c_{n}(T)$ and their derivatives with respect to $T, c_{n}^{\prime}(T)$, they provide a more sensitive test of the model than that obtained by considering the pressure alone. The expressions (15) can be read as Taylor series expansions with expansion coefficients

$$
\begin{aligned}
& \frac{e}{T^{4}}=\sum_{n} e_{n}(T)\left(\frac{\mu_{q}}{T}\right)^{n}, \quad e_{n}(T)=3 c_{n}(T)+c_{n}^{\prime}(T), \\
& \frac{s}{T^{3}}=\sum_{n} s_{n}(T)\left(\frac{\mu_{q}}{T}\right)^{n}, \quad s_{n}(T)=(4-n) c_{n}(T)+c_{n}^{\prime}(T) .
\end{aligned}
$$

Figure 3 shows a comparison of the QPM results for $e_{2,4}$ and $s_{2,4}$ [obtained through fine but finite difference approximations of the $c_{n}(T)$ ] with the corresponding lattice QCD results from Ref. [39]. The QPM parameters are the same as in Fig. 2, and the agreement with the lattice data is fairly good. The pronounced structures observed in the vicinity of the transition temperature are a result of the change in curvature of $G^{2}\left(T, \mu_{q}=0\right)$ at $T=T_{c}$ [see Eq. (9)]. Note that the derivatives $c_{n}^{\prime}(T)$ were estimated in Ref. [39] by finite difference approximations of the available lattice QCD results for $c_{n}(T)$. Adjusting the difference approximation in our QPM to the lattice procedure reproduces the pronounced structures in the vicinity of $T_{c}$ much better [43].

We close this section with a calculation of the quark number susceptibilities, which play a role in the calculation of event-by-event fluctuations of conserved quantities such as net baryon number, isospin, or electric charge [44-47]. Across the quark-hadron phase transition they are expected to become large. For instance, the peak structure in $c_{4}(T)$ [which for small $\mu_{B} / T$ gives the dominant $\mu_{B}$ dependence of $\chi_{B}$, see Eq. (14)] can be interpreted as an indication for critical behavior. Quark number susceptibilities have been evaluated in lattice QCD simulations by Gavai and Gupta [48], using constant bare quark masses $m_{q 0}=0.1 T_{c}$ with $T_{c}$ fixed by $m_{\rho} / T_{c}=5.4$. By introducing separate chemical potentials for $u$ and $d$ quarks and considering a simultaneous expansion of the QCD partition function $Z\left(T, \mu_{u}, \mu_{d}\right)$ in terms of $\mu_{u}$ and $\mu_{d}$, the leading $\mu_{u, d}$-independent contribution to the quark

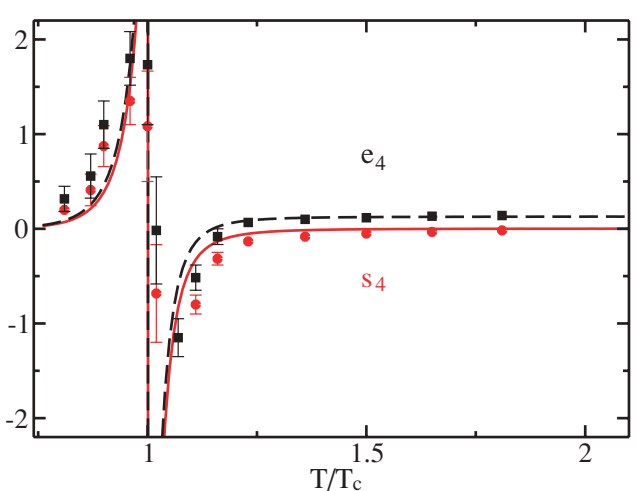

FIG. 3. (Color online) Comparison of the Taylor series expansion coefficients for $e_{n}(T)$ (squares/dashed black lines) and $s_{n}(T)$ (circles/solid red lines) for $N_{f}=2$ from Ref. [39] with the QPM (same parameters as in Fig. 2). Left panel: $n=2$. Right panel: $n=4$. For details see text. 


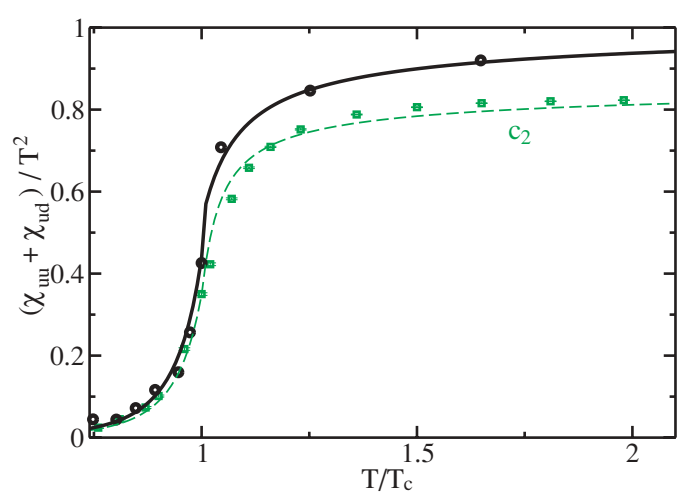

FIG. 4. (Color online) Comparison of the QPM result for $\left(\chi_{u u}+\right.$ $\left.\chi_{u d}\right) / T^{2}$ (solid line) with lattice QCD data (circles) from Ref. [48] for $N_{f}=2$, extrapolated to the continuum as suggested in Ref. [49]. The QPM parameters are $\lambda=7.0, T_{s}=0.76 T_{c}$, and $b=431$, with $T_{c}=175 \mathrm{MeV}$. For comparison, we also show lattice QCD data for $c_{2}(T)$ for $N_{f}=2$ from Ref. [41] (squares) together with the corresponding QPM fit (dashed line), using the same parameters as in Fig. 2.

number susceptibility $\chi_{q}=9 \chi_{B}$ can be expressed in terms of $\chi_{u u}, \chi_{u d}$, and $\chi_{d d}$, where

$$
\chi_{a b}=\left.\frac{\partial^{2} p\left(T, \mu_{u}, \mu_{d}\right)}{\partial \mu_{a} \partial \mu_{b}}\right|_{\mu_{a}=\mu_{b}=0} .
$$

These linear quark number susceptibilities can be related to the Taylor series expansions in Eqs. (11) and (14) through

$$
c_{2}(T)=\frac{1}{2 T^{2}}\left(\chi_{u u}+2 \chi_{u d}+\chi_{d d}\right) .
$$

For $m_{u}=m_{d}$ one finds $\chi_{u u}=\chi_{d d}$. In Fig. 4 we compare lattice QCD results [48] for $\left(\chi_{u u}+\chi_{u d}\right) / T^{2} \equiv c_{2}(T)$ with a QPM fit. The QPM parameters are adjusted to the lattice data from Ref. [48], after extrapolating the latter to the continuum by multiplying with a factor $d=0.465$ as advocated in Ref. [49]. For comparison, we also show $c_{2}(T)$ from Ref. [41] and the corresponding QPM parametrization from Fig. 2. Note that the latter data have not yet been extrapolated to the continuum. If we performed a continuum extrapolation of the $c_{2}(T)$ data from Ref. [41] by a factor $d=1.1$ for $T \geqslant T_{c}$ as in the case of $c_{0}(T)$ (cf. Fig. 1), both results would agree at large $T$ within $1 \%$. In the transition region some deviations would remain, owing to the different bare quark masses and actions employed in Refs. [41] and [48].

\section{Isentropic trajectories for $N_{f}=2$ quark flavors}

Ideal relativistic hydrodynamics [1-6] is considered to be the appropriate framework for describing the expansion of strongly interacting quark-gluon matter created in relativistic heavy-ion collisions. This approach requires approximate local thermal equilibrium and small dissipative effects. Because the fireballs created in heavy-ion experiments are small, pressure gradients are big, and expansion rates are large, thermalization must be maintained by sufficiently fast momentum transfer rates, resulting in microscopic thermalization time scales that are short compared to the macroscopic expansion time.
The hydrodynamic description remains valid as long as the particles' mean-free paths are much smaller than both the geometric size of the expanding fireball and its Hubble radius.

The hydrodynamic equations of motion result from the local conservation laws for energy-momentum and conserved charges, $\partial_{\mu} T^{\mu \nu}(x)=0$ and $\partial_{\mu} j_{i}^{\mu}(x)=0$. Here, $T^{\mu \nu}$ denotes the energy-momentum stress tensor and $j_{i}^{\mu}$ the four-current of conserved charge $i$ at space-time coordinate $x$. Heavy-ion collisions are controlled by the strong interaction, which conserves baryon number, isospin, and strangeness. If we assume zero net isospin and strangeness densities in the initial state, only the conservation of the baryon number four-current $j_{B}^{\mu}$ needs to be taken into account dynamically.

The ideal fluid equations are obtained by assuming locally thermalized momentum distributions, in which case $T^{\mu \nu}$ and $j_{B}^{\mu}$ take on the simple ideal fluid forms $T^{\mu \nu}=(e+p) u^{\mu} u^{\nu}-$ $p g^{\mu \nu}$ and $j_{B}^{\mu}=n_{B} u^{\mu}$ [50]. Here $g^{\mu \nu}$ is the Minkowski metric, $u^{\mu}(x)$ the local four-velocity of the fluid, and $e(x), p(x)$, and $n_{B}(x)$ denote the energy density, pressure, and baryon density in the local fluid rest frame, respectively. The resulting set of five equations of motion for six unknown functions is closed by the EoS that relates $p, e$, and $n_{B}$. This is where the lattice QCD data and our QPM parametrization of the lattice EoS enter the description of heavy-ion collision dynamics.

Once the initial conditions are specified, the further dynamical evolution of the collision fireball is entirely controlled by this EoS. Specifically, the accelerating power of the fluid (i.e., its reaction to pressure gradients, which provide the thermodynamic force driving the expansion) is entirely controlled by the (temperature-dependent) speed of sound, $c_{s}=\sqrt{\partial p / \partial e}$. To the extent that ideal fluid dynamics is a valid description and/or dissipative effects can be controlled, the observation of collective flow patterns in heavy-ion collisions can thus provide constraints on the EoS of the matter formed in these collisions.

Ideal fluid dynamics is entropy conserving; that is, the specific entropy $\sigma \equiv s / n_{B}$ of each fluid cell (where $s$ is the local entropy density) stays constant in its comoving frame. Although different cells usually start out with different initial specific entropies, and thus the expanding fireball as a whole maps out a broad band of widely varying $s / n_{B}$ values, each fluid cell follows a single line of constant $s / n_{B}$ in the $T\{-\} \mu_{B}$ phase diagram. It is therefore of interest to study the characteristics of such isentropic expansion trajectories, in particular the behavior of $p / e$ or $c_{s}^{2}=\frac{\partial p}{\partial e}$ along them.

The isentropic trajectories for different values of $s / n_{B}$ follow directly from the first-principles evaluation of the lattice EoS and its QPM parametrization considered in the previous section. For $N_{f}=2$ dynamical quark flavors, the truncated Taylor series expansions for baryon number and entropy density with expansion coefficients $c_{n}(T)$ and $s_{n}(T)$ according to Eqs. (17) were employed in Ref. [39] to determine the isentropic trajectories for $s / n_{B}=300,45$, and 30, sampling those regions of the phase diagram that can be explored with heavy-ion collisions at RHIC, SPS, and AGS/SIS300, respectively. To directly compare the QPM with these lattice results, we calculate $n_{B}$ from Eq. (13) and $s$ from Eqs. (15) and (16) up to $\mathcal{O}\left[\left(\mu_{B} / T\right)^{6}\right]$, where $c_{2,4,6}(T)$ are obtained from Eq. (12), $c_{0}(T)=p\left(T, \mu_{B}=0\right) / T^{4}$ from Eqs. (1) and (2), 
and the derivatives $c_{n}^{\prime}(T)$ are estimated through fine but finite difference approximations of the $c_{n}(T)$.

Besides investigating the impact of different continuum extrapolations of $c_{0}(T)$ on the pattern of isentropic trajectories, we can ask whether the differences observed between the parametrizations of $c_{0}(T)$ and $c_{2}(T)$ can be absorbed in such an extrapolation. Note that, even though the cutoff dependence of the lattice results is qualitatively similar at $\mu_{B}=0$ and at $\mu_{B} \neq 0$, no uniform continuum extrapolation is expected for the different Taylor expansion coefficients [41,51]. In Fig. 5 we show the raw lattice data for $c_{0}(T)$ [17] (squares) together with a continuum extrapolation (circles) obtained by multiplying the raw data for $T \geqslant T_{c}$ by a factor $d=1.1$. The corresponding QPM parametrizations [“fit 1" (dash-dotted) and "fit 2" (dashed) in the upper panel of Fig. 5] reproduce the lattice QCD results impressively well. Nonetheless, the corresponding QPM results for $c_{2,4}(T)$ underpredict the lattice data, as depicted in the bottom panel of Fig. 5. In particular, the pronounced structure in $c_{4}(T)$ at $T_{c}$ is not well reproduced
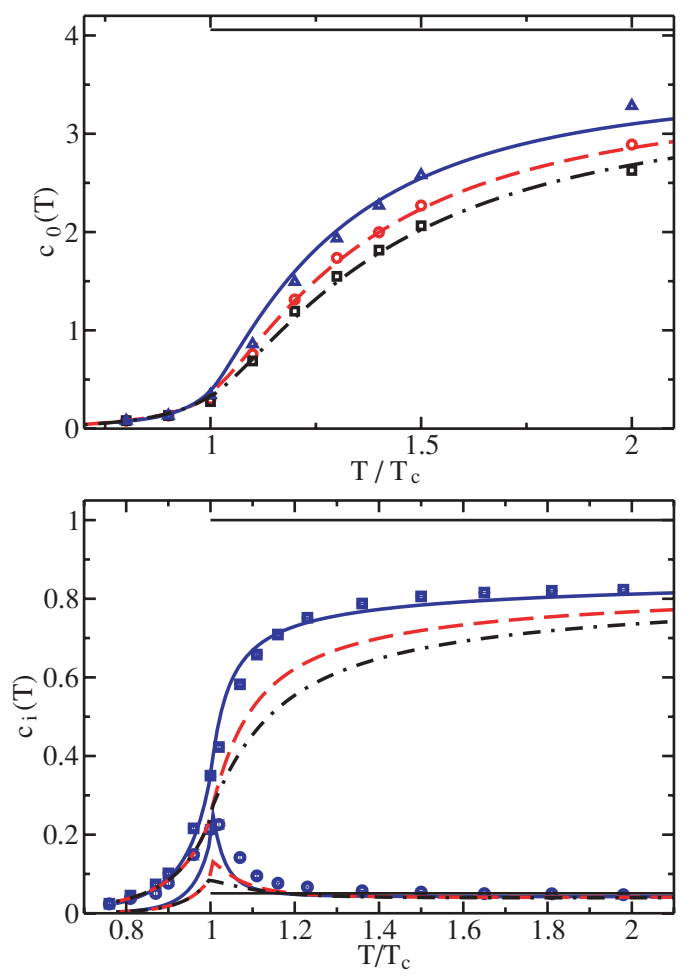

FIG. 5. (Color online) Top panel: $c_{0}(T)=p\left(T, \mu_{B}=0\right) / T^{4}$ as a function of $T / T_{c}$ for $N_{f}=2$. Raw lattice QCD data from Ref. [17] (squares) and guesses for the continuum extrapolated data obtained by multiplying (for $T \geqslant T_{c}=175 \mathrm{MeV}$ ) by a factor $d=1.1$ (circles) and $d=1.25$ (triangles) $[17,40]$ are shown together with the corresponding QPM fits (dashed-dotted, dashed, and solid curves, respectively). The QPM parameters read $B\left(T_{c}\right)=0.31 T_{c}^{4}, b=344.4, \lambda=2.7$, and $T_{s}=0.46 T_{c}$ for the dashed-dotted line ("fit 1 "); they are the same as in Fig. 1 for the dashed line ("fit 2") and the same as in Fig. 2 [with $B\left(T_{c}\right)=0.61 T_{c}^{4}$ ] for the solid line ("fit 3"). Bottom panel: Corresponding QPM results compared with lattice results for $c_{2}(T)$ (squares) and $c_{4}(T)$ (circles) as a function of $T / T_{c}$ with the same line code as in the top panel. The horizontal lines indicate the Stefan-Boltzmann values. by the QPM fit. If we instead use a QPM parametrization that optimally reproduces $c_{2}(T)$ (solid line in the bottom panel of Fig. 5), the corresponding QPM result for $c_{0}(T)$ ("fit 3" in the upper panel of Fig. 5) agrees fairly well with an assumed continuum extrapolation of the raw lattice data by a factor $d=1.25$ for $T \geqslant T_{c}$ (triangles).

In Fig. 6, the QPM results for $s / n_{B}=300$ and 45 employing different fits are exhibited together with the results of Ref. [39]. In the left panel of Fig. 6 we see that the lattice results can be fairly well reproduced when two separately optimized QPM parametrizations for $c_{0}(T)$ and $c_{2}(T)$ (cf. Fig. 1 and 2) are used simultaneously. This approach, however, would give up thermodynamic consistency of the model. When a single consistent parametrization for both $c_{0}$ and $c_{2}$ is used, specifically the one shown by the solid lines in Fig. 5 corresponding to "fit 3," the QPM produces the isentropes shown in the right panel of Fig. 6. (The other two fits shown in Fig. 5 yield almost the same isentropic expansion trajectories as "fit 3"). For large $s / n_{B}$ (i.e., for small net baryon densities), differences between the QPM results in the left and right panels of Fig. 6 are small, although the left fit shows a weak structure near $T_{c}$ that disappears in the self-consistent fit shown in the right panel. With decreasing $s / n_{B}$ the differences between the results from the two fitting strategies increase. They are mainly caused by differences in the slope of $c_{0}(T)$, which affect the shape of $s(T) / T^{3}$ and translate, for a given isentropic trajectory, into large variations of $\mu_{B}$ near $T_{c}\left(\mu_{B}=0\right)=$ $175 \mathrm{MeV}$ while causing only small differences of about $6 \%$ at large $T$. In particular, the pronounced structures of the isentropic trajectory near the estimated phase border are completely lost in the self-consistent fit procedure. This shows that the pattern of the isentropic expansion trajectories is quite sensitive to details of the EoS. For instance, when employing $c_{0}(T)$ data, which were extrapolated to the continuum by multiplication with a factor $d=1.25$ at $T \geqslant T_{c}$ while leaving $c_{2,4,6}(T)$ unchanged, one obtains the isentropic expansion trajectories shown by open squares in the right panel of Fig. 6 , which also lack any structure near the phase transition.

Changing the deconfinement transition temperature to $T_{c}=$ $170 \mathrm{MeV}$ results in a shift of the trajectories by about $10 \%$ in the $\mu_{B}$ direction near $T_{c}$ but has negligible consequences for $T \geqslant 1.5 T_{c}$. At asymptotically large $T$, where $c_{0,2}(T)$ are essentially flat, the relation $\frac{\mu_{B}}{T}=18 \frac{c_{0}}{c_{2}}\left(\frac{n_{B}}{s}\right)$ holds for small $\mu_{B}$ (i.e., lines of constant specific entropy are essentially given by lines of constant $\left.\mu_{B} / T\right)$, as is the case in a quark-gluon plasma with perturbatively weak interactions.

Figure 6 also shows the chemical freeze-out points deduced from hadron multiplicity data for $\mathrm{Au}+\mathrm{Au}$ collisions at $\sqrt{s}=130 \mathrm{~A} \mathrm{GeV}$ at RHIC $\left(T_{\text {chem }}=169 \pm 6 \mathrm{MeV}\right.$ and $\mu_{B, \text { chem }}=38 \pm 4 \mathrm{MeV} \mathrm{[52])} \mathrm{and} \mathrm{for} 158 A \mathrm{GeV} \mathrm{Pb}+\mathrm{Pb}$ collisions at the CERN SPS $\left(T_{\text {chem }}=154.6 \pm 2.7 \mathrm{MeV}\right.$ and $\mu_{B, \text { chem }}=245.9 \pm 10.0 \mathrm{MeV}$ [53]). Note that the specific entropies at these freeze-out points as deduced from the statistical model [55] are $s / n_{B}=200$ for RHIC-130 and $s / n_{B}=30$ for SPS-158 (i.e., only about $2 / 3$ of the values corresponding to the QPM fit of the QCD lattice data). One should remember, though, that the phenomenological values are deduced from experimental data using a complete spectrum of hadronic resonances whereas the lattice simulations were 

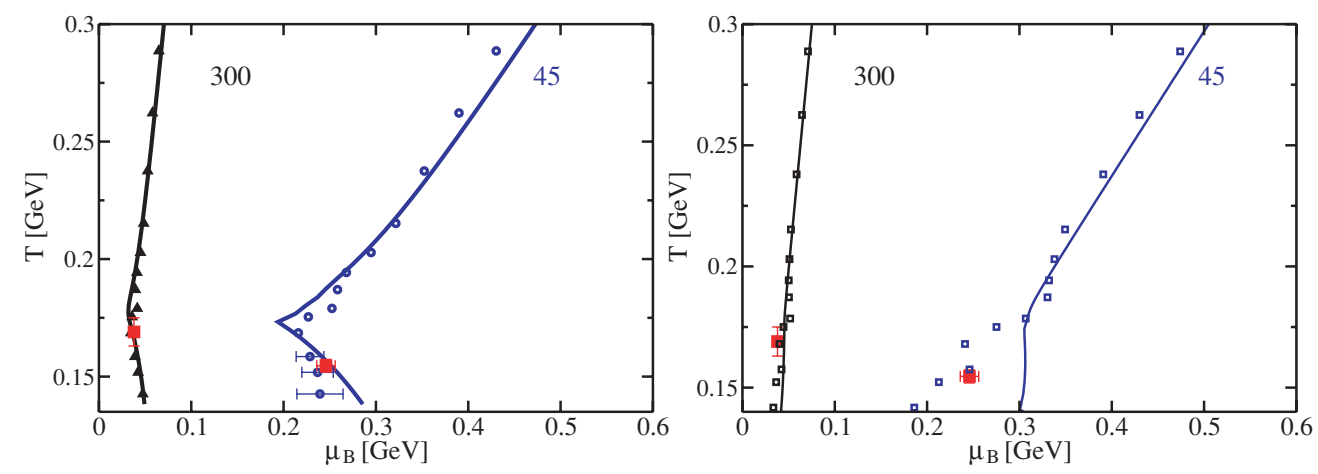

FIG. 6. (Color online) Isentropic evolutionary paths. Triangles and circles indicate $N_{f}=2$ lattice QCD data from Ref. [39] for $s / n_{B}=$ 300 and 45, respectively. Corresponding QPM results are depicted in the left panel for a mixed fit where $c_{0}(T)$ and $c_{2}(T)$ were fitted independently (cf. Figs. 1 and 2). In the right panel we show results from "fit 3" from Fig. 5, with open squares indicating the corresponding continuum-extrapolated lattice results where the raw $c_{0}(T)$ lattice data were multiplied by a constant factor $d=1.25$ at $T \geqslant T_{c}$ [17]. Full red squares show chemical freeze-out points deduced in Refs. [52,53] from hadron multiplicity data, as summarized in Ref. [54].

performed for only $N_{f}=2$ dynamical quark flavors with not quite realistic quark masses.

Figure 7 shows that along isentropic expansion lines the EoS is almost independent of the value of $s / n_{B}$. Accordingly, the speed of sound $c_{s}^{2}=\partial p / \partial e$ (which controls the buildup of hydrodynamic flow) is essentially independent of the specific entropy. Note that whether we employ the mixed fit or the thermodynamically consistent fits 1, 2, and 3 of Fig. 5 does not significantly affect the EoS along the isentropes; for large energy densities $e \gtrsim 30 \mathrm{GeV} / \mathrm{fm}^{3}$ the differences in $p(e)$ are less than $2 \%$.

\section{A remark on the QCD critical point}

At a critical point (CP) a first-order phase transition line terminates and the transition becomes second order.

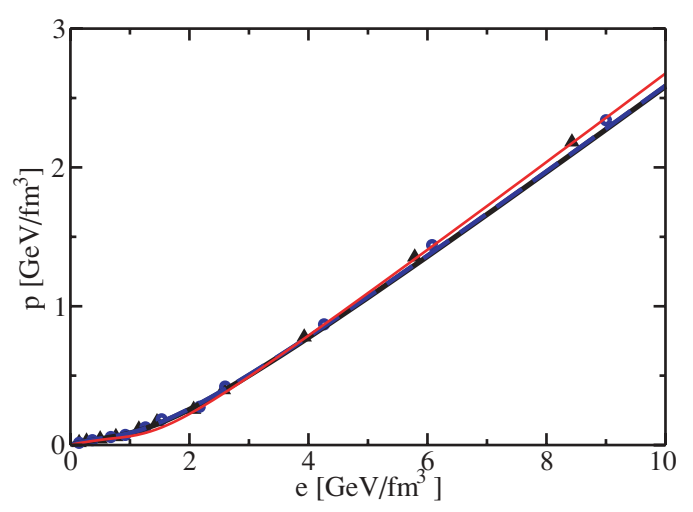

FIG. 7. (Color online) Lattice QCD data [39] of $p$ as a function of $e$ for $N_{f}=2$ along isentropes with $s / n_{B}=300$ (triangles) and 45 (circles), compared with the corresponding QPM results (solid blue and dashed black lines, respectively). These two thick lines employ the mixed fit shown in the left panel of Fig. 6 and are indistinguishable for $s / n_{B}=300$ and 45 . The thin solid lines show corresponding results for the self-consistent "fit 3" from Fig. 5. Again the curves for different $s / n_{B}$ are indistinguishable, and also the deviations from the mixed fit are minor.
QCD with $N_{f}=2+1$ dynamical quark flavors with physical masses is a theory where such a $\mathrm{CP}$ is expected at finite $T$ and $\mu_{B}$ [56-58]. Its precise location is still a matter of debate [48,59-61], but Fodor and Katz [59] claim $T_{E}=$ $162 \mathrm{MeV}$ and $\mu_{B, E}=360 \mathrm{MeV}$ for the critical values. In the following, we focus on initial baryon densities $n_{B}<$ $0.5 \mathrm{fm}^{-3}$, which, under the assumption of isentropic expansion with conserved $s / n_{B}=250$, corresponds to a baryon chemical potential $\mu_{B}(T=170 \mathrm{MeV})<60 \mathrm{MeV}$. This is sufficiently far from the conjectured $\mathrm{CP}$ that we should be justified in assuming that the EoS is adequately parametrized by our QPM for describing bulk thermodynamic properties and the hydrodynamical evolution of the hot QCD matter.

\section{EQUATION OF STATE}

In this section we concentrate on the physical case of $N_{f}=$ $2+1$ dynamical quark flavors and match the QPM fit to the lattice QCD data at temperatures above $T_{c}$ to a realistic hadron resonance gas EoS below $T_{c}$. In this way we construct an EoS that can be applied to all stages of the hydrodynamic expansion of the hot matter created in relativistic heavy-ion collisions at RHIC and LHC. We focus our attention on the region of small net baryon density explored at these colliders.

\section{A. Pressure as a function of energy density}

Our goal is to arrive at an $\operatorname{EoS}$ in the form $p\left(e, n_{B}\right)$ as needed in hydrodynamic applications. We anchor our QPM approach above $T_{c}$ to lattice QCD simulations for $N_{f}=2+1$ dynamical quark flavors presented in Refs. [17,62,63], where $p(T) / T^{4}$ and $e(T) / T^{4}$ were calculated by using $m_{q 0}=0.4 T$ and $m_{s 0}=T$. Unfortunately, Taylor series expansions for nonzero $\mu_{B}$ analogous to the $N_{f}=2$ case are not available for $N_{f}=2+1$. Effects of finite $\mu_{B}$ were studied in Ref. [64] for $N_{f}=2+1$ with the multiparameter reweighting method and successfully compared with the quasiparticle model in Ref. [37] by testing the extrapolation via Eq. (8). We here concentrate on results from lattice QCD 

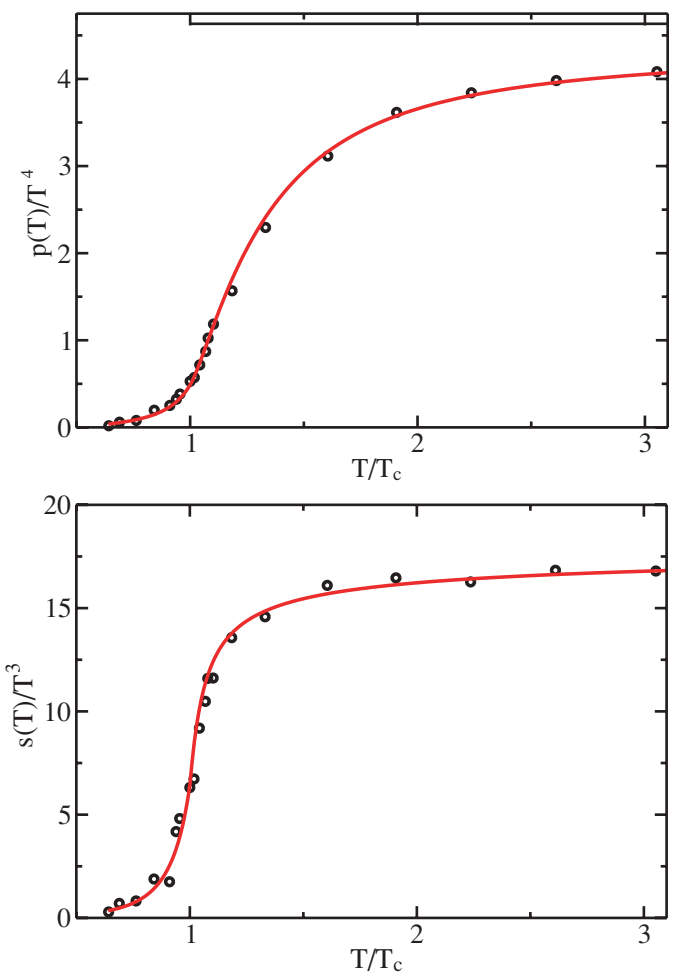

FIG. 8. (Color online) Comparison of the QPM with lattice QCD results (symbols) for the scaled pressure $p / T^{4}$ (top panel) and the scaled entropy density $s / T^{3}$ (bottom panel) as a function of $T / T_{c}$ for $N_{f}=2+1$ and $\mu_{B}=0$. The lattice QCD data [63] are already continuum extrapolated. The QPM parameters read $\lambda=7.6, T_{s}=$ $0.8 T_{c}, b=348.72$, and $B\left(T_{c}\right)=0.52 T_{c}^{4}$, where $T_{c}=170 \mathrm{MeV}$. In the top panel, the horizontal line indicates the Stefan-Boltzmann value $p_{\mathrm{SB}} / T^{4}=\bar{c}_{0}=\left(32+21 N_{f}\right) \pi^{2} / 180$, using $N_{f}=2.5$ to account for the nonzero strange quark mass.

simulations employing improved actions [17], which strongly reduce lattice discretization errors at high temperatures. First, we focus on the available data at $\mu_{B}=0$ and assume that the extension to nonzero $\mu_{B}$ can be accomplished through the QPM without any complications, relying on the successful test of our model at finite baryon density for $N_{f}=2$, as reported in the preceding section and earlier publications.

In Fig. 8 we compare the QPM results for the pressure $p(T) / T^{4}$ and entropy density $s(T) / T^{3}$ with $N_{f}=2+1$ lattice QCD data, where $s$ follows simply from $e$ and $p$ through $s / T^{3}=(e+p) / T^{4}$. The parametrization found at $\mu_{B}=0$ is now used to obtain the required thermodynamic observables at nonzero $n_{B}$ from the full QPM via Eqs. (1) and (6) and the relation $e+p-T s=\mu_{B} n_{B}$, exploiting the Maxwell relation (8).

In Fig. 9 we compare the QPM equation of state $p(e)$ at $n_{B}=0$ with the corresponding lattice QCD result deduced from data for $p$ and $e$ at $n_{B}=0$ [17] in the energy density domain explored by heavy-ion collisions at RHIC. The lattice data used [17] were already extrapolated to the continuum in Ref. [63]. In Refs. [62,65] $T_{c}=(173 \pm 8) \mathrm{MeV}$ was found for the deconfinement transition temperature. Recent analyses [66, 67] have pointed out remaining uncertainties in the extraction
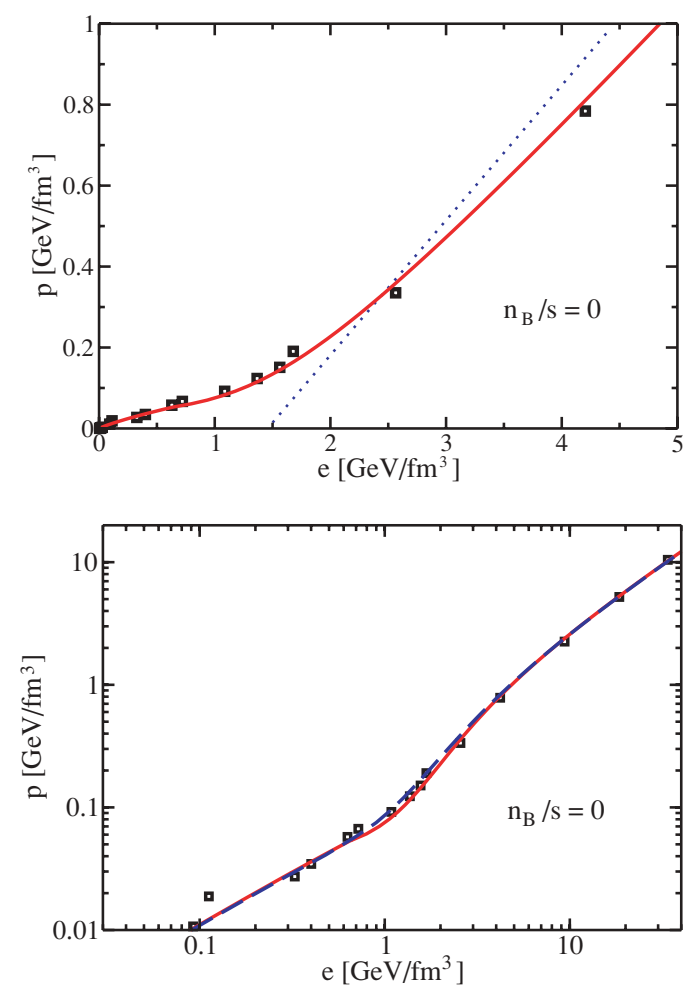

FIG. 9. (Color online) Top panel: $N_{f}=2+1$ QPM equation of state of strongly interacting matter for vanishing net baryon density (solid line) compared with $N_{f}=2+1$ continuum-extrapolated lattice QCD data [63] (squares) at $n_{B}=0$. The dotted line represents $p(e)$ for a gas of massless noninteracting quarks and gluons with a bag constant $B^{1 / 4}=230 \mathrm{MeV}$. Bottom panel: QPM EoS for $N_{f}=2$ (dashed line) employing "fit 2" in Figs. 1 and 5, compared with lattice data [63] (squares) and QPM results (solid line) for $N_{f}=2+1$, in logarithmic representation.

of $T_{c}$ that would have to be sorted out by simulations on larger lattices. Here, we set the physical scale to $T_{c}=170 \mathrm{MeV}$ (see the following discussion). In the transition region the energy density $e(T)$ varies by $300 \%$ within a temperature interval of $\Delta T \approx 20 \mathrm{MeV}$ whereas $p(T)$ rises much more slowly (see upper panels in Figs. 8 and 9). This indicates a rapid but smooth crossover for the phase transition from hadronic to quark matter. At large energy densities $e \geqslant 30 \mathrm{GeV} / \mathrm{fm}^{3}$ the EoS follows roughly the ideal gas relation $e=3 p$. For the sake of comparison, a bag model equation of state describing a gas of massless noninteracting quarks and gluons with bag constant $B^{1 / 4}=230 \mathrm{MeV}$ is also shown in Fig. 9 (straight dotted line in the top panel).

As an aside, differences in $p\left(e, n_{B}=0\right)$ arising from considering different numbers $N_{f}$ of dynamical quark flavors are investigated in the bottom panel of Fig. 9. Comparing the QPM result for $N_{f}=2+1$ with the result for $N_{f}=2$ (see Fig. 1), we see that the latter exceeds the $N_{f}=2+1$ result in the transition region (by about $12 \%$ at $e=1 \mathrm{GeV} / \mathrm{fm}^{3}$ ). For larger energy densities $e \geqslant 3 \mathrm{GeV} / \mathrm{fm}^{3}$ the EoS is found to be fairly independent of $N_{f}$ even though at fixed $T$ both $p(T)$ and $e(T)$ are significantly smaller for $N_{f}=2$ than for $N_{f}=2+1$ (see Figs. 1 and 8). 


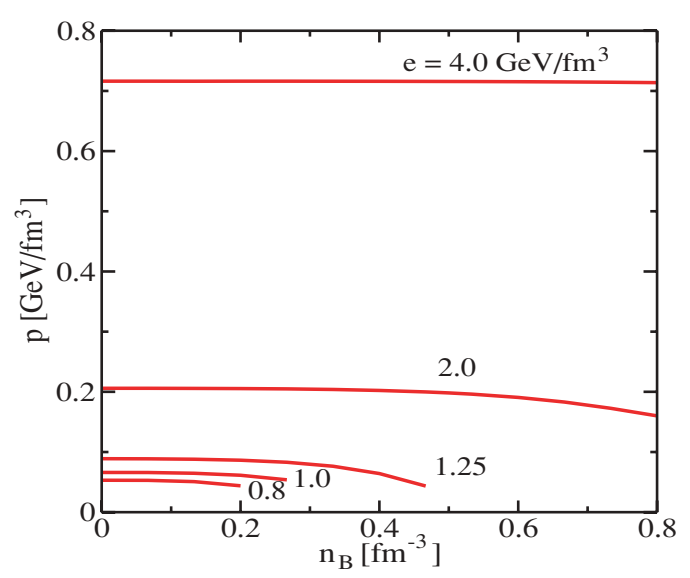

FIG. 10. (Color online) Baryon number density dependence of the $\operatorname{EoS} p\left(e, n_{B}\right)$ at constant energy density $e$ as indicated. The curves end where the solution of the flow equation (8) is no longer unique.

\section{B. Baryon density effects}

We turn now to the baryon density dependence of the EoS. Since for hydrodynamics the relation $p\left(e, n_{B}\right)$ matters, we consider the $n_{B}$ dependence of the pressure at fixed energy density. Figure 10 shows that significant baryon density dependence of the pressure at fixed energy density arises only for $e \leqslant 2 \mathrm{GeV} / \mathrm{fm}^{3}$. At the smallest energy densities considered here, the dependence of $p$ on $n_{B}$ cannot be determined over the entire $n_{B}$ region shown since the flow equation (8) for $G^{2}\left(T, \mu_{B}\right)$ has no unique solution at large $\mu_{B}$ for temperatures far below the estimated transition temperature $T_{c}\left(\mu_{B}\right)$ [38]. However, in the family of equations of state that we will construct and employ in the following, this peculiar feature for small $e$ will not occur. Larger baryon densities, which become relevant at AGS and CERN/SPS energies or the future CBM project at the FAIR/SIS300 facility, deserve separate studies. Under RHIC and LHC conditions finite baryon density effects on the EoS can be safely neglected at all energy densities for which the QPM can be applied.

\section{Robustness of the QPM $\operatorname{EoS} p\left(e, n_{B} \approx 0\right)$}

We now perform a naive chiral extrapolation of the QPM EoS by setting $m_{q 0}=0$ and $m_{s 0}=150 \mathrm{MeV}$ in the thermodynamic expressions, leaving all other parameters fixed. The resulting EoS is shown in the upper left panel of Fig. 11. In this procedure a possible dependence of the QPM parameters in Eqs. (9) and (10) and, especially, of the integration constant $B\left(T_{c}\right)$ in Eq. (1) on the quark mass parameters $m_{a 0}$ is completely neglected. Note that in the transition region $\left(e \sim 1 \mathrm{GeV} / \mathrm{fm}^{3}\right)$ the chirally extrapolated result exceeds the original QPM EoS (which was fitted to lattice data with unphysical quark masses) by approximately $10 \%$. For higher energy densities $e \geqslant 2 \mathrm{GeV} / \mathrm{fm}^{3}$ these quark mass effects are seen to be negligible.

For $e \leqslant 0.45 \mathrm{GeV} / \mathrm{fm}^{3}$, the fat solid line in the upper left panel of Fig. 11 shows a hadron resonance gas model EoS with a physical mass spectrum in chemical equilibrium [68]. Obviously, it exceeds both the lattice QCD data and their

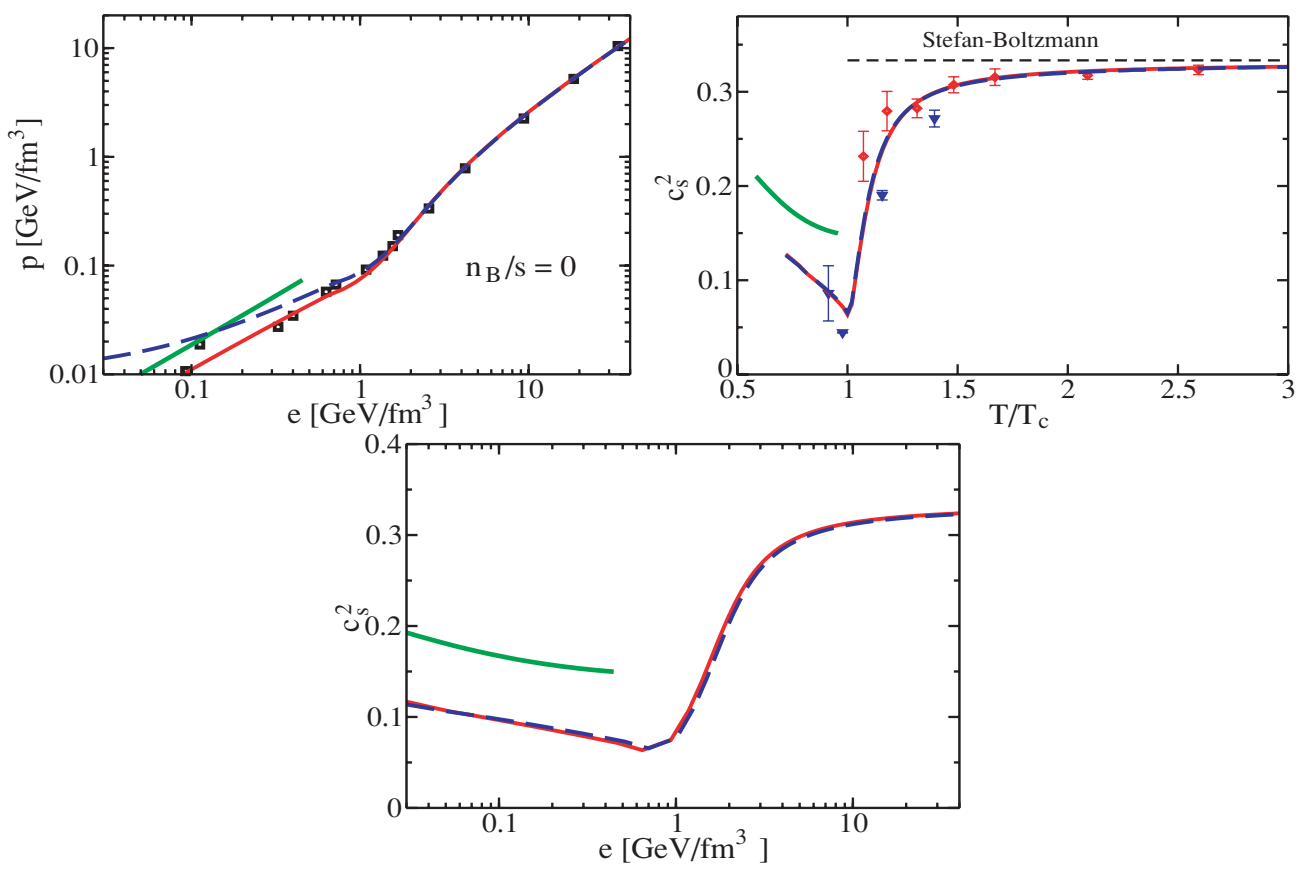

FIG. 11. (Color online) Upper left panel: QPM EoS for $N_{f}=2+1$ (solid red) and its chiral extrapolation to physical quark masses (dashed blue). Squares show LQCD data for $N_{f}=2+1$ quark flavors with unphysical masses [63]. Upper right panel: Comparison of the squared speed of sound, $c_{s}^{2}=\partial p / \partial e$, as a function of $T / T_{c}$ from the QPM with lattice QCD data [69] (diamonds and triangles) deduced from the $N_{f}=2$ data for $p(e)$ in Ref. [39]. Differences between the QPM fit to the LQCD data (solid red) and its extrapolation to physical quark masses (dashed blue) for $N_{f}=2+1$ are almost invisible. Bottom panel: Same as upper right panel, but plotted as a function of energy density $e$. In all three panels the solid green line shows the hadron resonance gas model EoS "aa1" from Ref. [68]. 
QPM parametrization. The chirally extrapolated QPM EoS, however, approaches and intersects the hadron resonance gas EoS.

Considering $p / e$ as a function of $e$, we find for the lattice-fitted QPM EoS a softest point $(p / e)_{\min }=0.075$ at $e_{c}=$ $0.92 \mathrm{GeV} / \mathrm{fm}^{3}$. For the chirally extrapolated QPM EoS, the softest point moves slightly upward to $(p / e)_{\min }=0.087$ at $e_{c}=1.1 \mathrm{GeV} / \mathrm{fm}^{3}$, in good agreement with the lattice QCD data, which show a softest point $(p / e)_{\min }=0.080$ at $e_{c}=$ $1 \mathrm{GeV} / \mathrm{fm}^{3}$.

The small differences between the lattice-fitted QPM equation of state and its chirally extrapolated version for $N_{f}=2+1$ can be further analyzed by studying the squared speed of sound, $c_{s}^{2}$. In the upper right panel of Fig. $11, c_{s}^{2}$ is shown as a function of $T / T_{c}$ for both versions of the QPM EoS and compared with lattice QCD results [69]. One sees that, as far as $c_{s}^{2}$ is concerned, the extrapolation of the QPM to physical quark masses has no discernible consequences, and both versions of the QPM EoS therefore have identical driving power for collective hydrodynamic flow. Hydrodynamically it is thus of no consequence that the available lattice QCD data for the EoS were obtained with unphysical quark masses.

The EoS is also fairly robust against variations in the particular choice of the physical scale $T_{c}$. In Fig. 12 we show $p(e)$ when setting $T_{c}=160,170$, and $180 \mathrm{MeV}$, respectively, thereby covering the "reasonable range" advocated in Refs. [62,65]. For small energy densities and, in particular, for large $e \geqslant 5 \mathrm{GeV} / \mathrm{fm}^{3}$ the EoS is rather independent of the choice of the value for $T_{c}$. At intermediate $e, p(e)$ varies at most by $\pm 20 \%$ for $\Delta T_{c}= \pm 10 \mathrm{MeV}$. As discussed in Sec. III D, we must bridge over this intermediate region when interpolating between the QPM and hadron resonance EoS, so this weak dependence on the physical scale $T_{c}$ is irrelevant in practice.

Next we examine variations in $p\left(e, n_{B} \approx 0\right)$ arising from different continuum extrapolations of the lattice QCD data. Considering the various "by hand" continuum extrapolations of $p(T) / T^{4}$ shown in Fig. 5 for $N_{f}=2$, we plot the resulting EoS in Fig. 13. Again, some weak sensitivity is observed only in the transition region, which will be bridged over in the next section by matching the QPM EoS to a realistic hadron resonance gas below $T_{c}$. The problem discussed in Sec. II B,

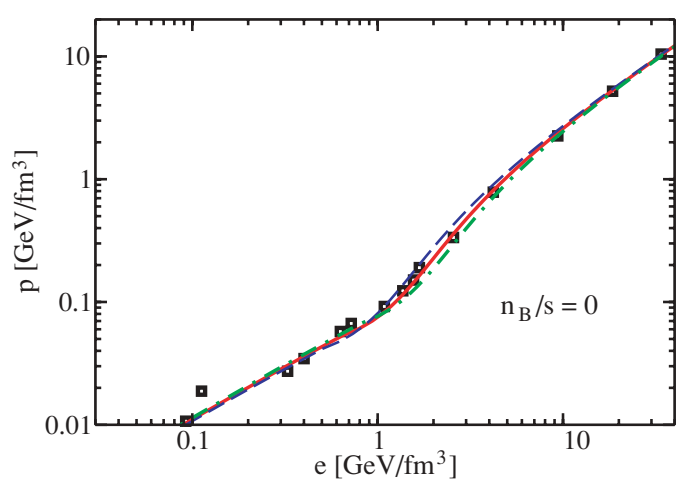

FIG. 12. (Color online) Dependence of the EoS for $N_{f}=2+1$ on the chosen value of the physical scale $T_{c}$. Dashed, solid, and dash-dotted curves correspond to $T_{c}=160,170$, and $180 \mathrm{MeV}$, respectively. Lattice data (squares) are from Ref. [63].

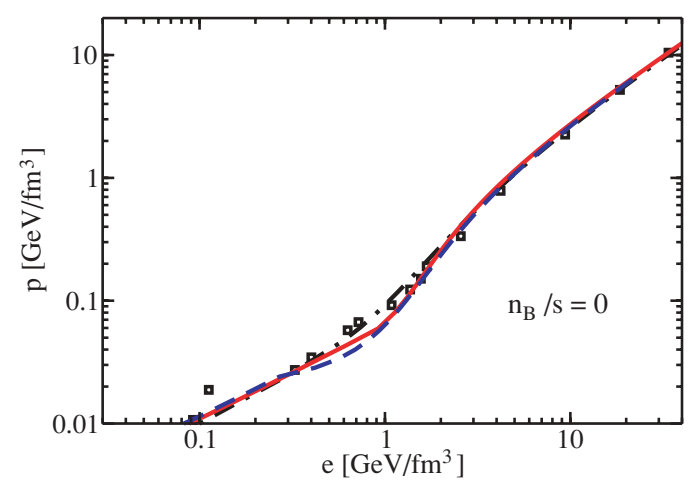

FIG. 13. (Color online) Dependence of the EoS for $N_{f}=2$ on the employed continuum extrapolation as performed in Fig. 5. Dash-dotted, dashed, and solid curves correspond to the QPM parametrizations of the raw lattice QCD data [17] and continuum extrapolations of these data by a factor $d=1.1$ and $d=1.25$, respectively.

that different optimum QPM parameters are found by fitting the model to $c_{0}(T)$ or $c_{2}(T)$ (see Figs. 1, 2, and 5), does not matter here since the differences in the resulting parametrizations manifest themselves only weakly in the $\operatorname{EoS} p(e)$ and are completely negligible for $e>5 \mathrm{GeV} / \mathrm{fm}^{3}$. In the transition region near $e \approx 1 \mathrm{GeV} / \mathrm{fm}^{3}$ the resulting uncertainties are of order $20 \%$ (see Fig. 13), but again the interpolation to the hadronic EoS largely eliminates this remaining sensitivity.

We close this section by exploring the robustness of the $\operatorname{EoS} p(e)$ against variations among different existing lattice QCD simulations resulting from present technical limitations. In doing so we keep in mind the aforementioned negligibly small baryon density effects in the region $n_{B}<0.5 \mathrm{fm}^{-3}$. In the top panel of Fig. 14 we show the available lattice QCD results for $p(T) / T^{4}$ with $N_{f}=2+1$ dynamical quark flavors from three different groups $[63,70,71]$ and compare them with our QPM adjusted individually to each of these data sets. The differences among the data sets reflect the use of different lattice actions, lattice spacings, bare quark masses, etc. As shown in the figure, these differences can be absorbed by the QPM through slight variations in the fit parameters. However, when presenting the lattice results and corresponding QPM fits in the form of an $\operatorname{EoS} p(e)$, they all coincide for $e \geqslant 5 \mathrm{GeV} / \mathrm{fm}^{3}$ (bottom panel of Fig. 14). (The agreement is excellent up to $e \approx$ $30 \mathrm{GeV} / \mathrm{fm}^{3}$ whereas at higher energy densities a small difference of about $6 \%$ between the equations of state from Refs. [63] and [71] begins to become visible.) In this region the EoS can be parameterized linearly by $p=\alpha e+\beta$ with $\alpha=0.310 \pm 0.005$ and $\beta=-(0.56 \pm 0.07) \mathrm{GeV} / \mathrm{fm}^{3}$. This robustness of the lattice QCD EoS for $e \geqslant 5 \mathrm{GeV} / \mathrm{fm}^{3}$ implies that it can be considered as stable input for hydrodynamic simulations of heavy-ion collisions and that the EoS is well constrained at high energy densities where the QPM EoS (which incorporates the lattice data) significantly differs from and improves upon the bag model EoS. However, the bottom panel of Fig. 14 shows that significant uncertainty remains in the immediate vicinity of the phase transition [owing to the steep rise of $e(T)$ near $T_{c}$ the region $0.5<e<4 \mathrm{GeV} / \mathrm{fm}^{3}$ corresponds to only a narrow temperature interval around $T_{c}$ ] 

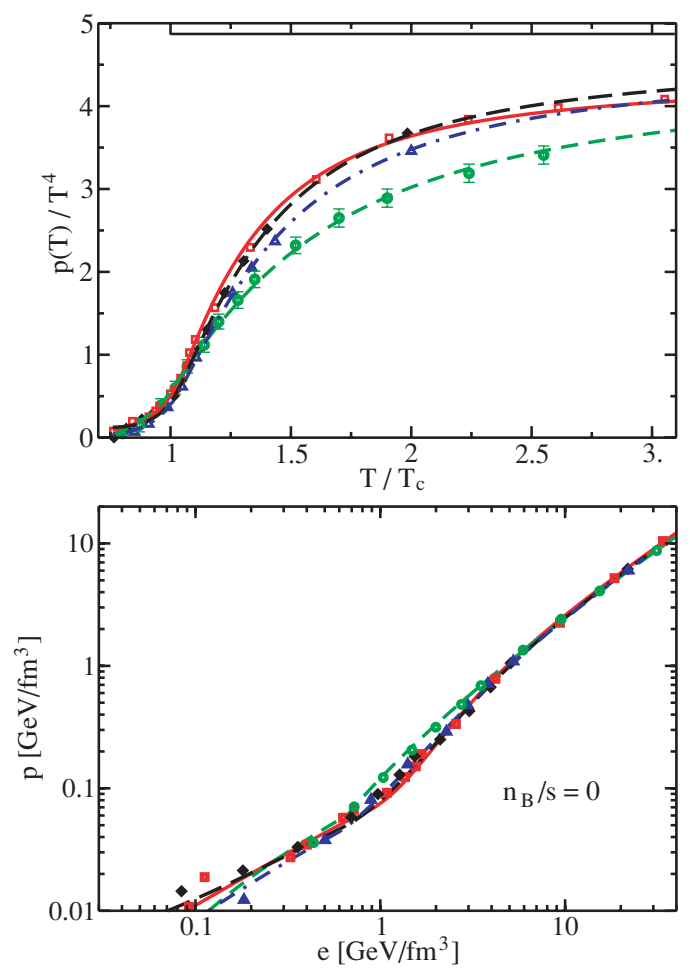

FIG. 14. (Color online) Stability of the QPM EoS fitted to lattice QCD results for $N_{f}=2+1$. Top panel: The scaled pressure $p(T) / T^{4}$ at $\mu_{B}=0$ from different lattice QCD calculations (Ref. [63] (squares), Ref. [70] (diamonds and triangles), and Ref. [71] (circles)), together with corresponding QPM fits (solid, long-dashed and dashdotted, and short-dashed lines, respectively). The fit parameters are optimized separately in each case, keeping, however, $B\left(T_{c}\right)=0.52 T_{c}^{4}$ with $T_{c}=170 \mathrm{MeV}$ in all four parametrizations fixed. Bottom panel: The $\operatorname{EoS} p\left(e, n_{B}=0\right)$ corresponding to the data and fits shown in the top panel.

and that further improvements in the lattice QCD data are welcome to resolve this remaining ambiguity.

\section{Matching lattice QCD to a hadron resonance gas equation of state via the QPM}

In this section we will now match the lattice QCD EoS at high energy densities with a realistic hadron resonance gas model at low energy densities [72,73]. Because available lattice QCD simulations still employ unrealistic quark masses whereas the hadron gas model builds upon the measured spectrum of hadronic resonances, we will use the QPM to parametrize the lattice QCD EoS and extrapolate it to physical quark masses. Such quark mass effects matter most at the lower end of the temperature range covered by the lattice QCD data, which is, however, also the region where the transition from the QPM to the hadron resonance gas model must be implemented.

In the vicinity of the phase transition, the conditions of the lattice QCD evaluations in Refs. [17,39] correspond to a pion mass $m_{\pi} \approx 770 \mathrm{MeV}$. This large pion mass reduces the pressure at small energy density below that of a realistic hadron resonance gas. Smaller quark masses are necessary to properly account for the partial pressure generated by the light pion modes and their remnants in the temperature region around $T_{c}$. Nonetheless, the hadron resonance gas model has been shown to be consistent with the QCD lattice data below $T_{c}$ if one appropriately modifies its mass spectrum for consistency with the employed lattice parameters [63]. We will therefore adopt the hadron resonance gas model with physical mass spectrum $[72,73]$ as an appropriate approximation of the hadronic phase [74] and use the QPM to parametrize the lattice QCD EoS near and above $T_{c}$.

For the hadron resonance gas EoS [72,73] we use the implementation developed for the $(2+1)$-dimensional hydrodynamic code package AZHYDRO [68], which provides this EoS in tabulated form on a grid in the $\left(e, n_{B}\right)$ plane. Specifically, we use EoS "aa1" from the OSCAR Web site [68] up to $e_{1}=0.45 \mathrm{GeV} / \mathrm{fm}^{3}$. It describes a thermalized, but chemically nonequilibrated hadron resonance gas, with hadron abundance yield ratios fixed at all temperatures at their chemical equilibrium values at $T=T_{c}=170 \mathrm{MeV}$, as found empirically [75] in $\mathrm{Au}+\mathrm{Au}$ collisions at RHIC.

As seen in Fig. 11, the pressure $p(e)$ of the hadron resonance gas EoS does not join smoothly to that of the QPM EoS at $T_{c}$ (i.e., at $e_{1}=0.45 \mathrm{GeV} / \mathrm{fm}^{3}$ ), irrespective of whether one uses directly the QPM fit to the lattice QCD data with unphysical quark masses (solid red line in Fig. 11) or extrapolates the QPM to physical quark masses (dashed blue line). A thermodynamically consistent treatment thus requires a Maxwell-like construction, equating the two pressures at a common temperature $T_{c}$ and baryon chemical potential $\mu_{B}$. We opt here for a slightly different approach, which has the advantage of allowing a systematic exploration of the effects of details (e.g., stiffness or velocity of sound) of the EoS near $T_{C}$ on hydrodynamic flow patterns: We interpolate $p\left(e, n_{B}\right)$ at fixed baryon density $n_{B}$ linearly between the hadron resonance gas ("aa1") value at $e=e_{1}$ to its value in the QPM at a larger value $e_{m}$, keeping $e_{1}$ fixed but letting the "matching point" value $e_{m}$ vary. In our procedure $T\left(e_{m}\right) \geqslant T\left(e_{1}\right)$, so $T(e)$ is also interpolated linearly, as is the baryon chemical potential $\mu_{B}(e)$ at fixed $n_{B}$. [This is a convenient pragmatic procedure to interpolate the special tabular forms of the EoS between $e_{1}$ and $e_{m}$ employed in the following. Complete thermodynamic consistency would require involved polynomials for temperature and chemical potential interpolation. We utilize the linearized structures since the hydrodynamical evolution equations do not explicitly refer to $T$ and $\mu_{B}$ in the interpolation region; instead, only $p\left(e, n_{B}\right)$ matters.]

This produces a family of equations of state whose members are labeled by the matching point energy density $e_{m}$. We here explore the range $1.0 \leqslant e_{m} \leqslant 4.0 \mathrm{GeV} / \mathrm{fm}^{3}$ (see Fig. 15). Since the chiral extrapolation of the QPM fit to physical quark masses significantly affects the $\operatorname{EoS} p(e)$ only at energy densities below $1 \mathrm{GeV} / \mathrm{fm}^{3}$ (see upper left panel in Fig. 11), it does not matter whether we use for this procedure the direct QPM fit to the lattice QCD data or its chiral extrapolation.

Figure 15 shows the result for four selected $e_{m}$ values, $e_{m}=1.0,1.25,2.0$, and $4.0 \mathrm{GeV} / \mathrm{fm}^{3}$ (from bottom to top). For $e_{m}=3.0 \mathrm{GeV} / \mathrm{fm}^{3}$ one obtains a curve $p(e)$ (not shown) that extrapolates the hadron resonance gas with constant slope all the the way to the QPM curve. 

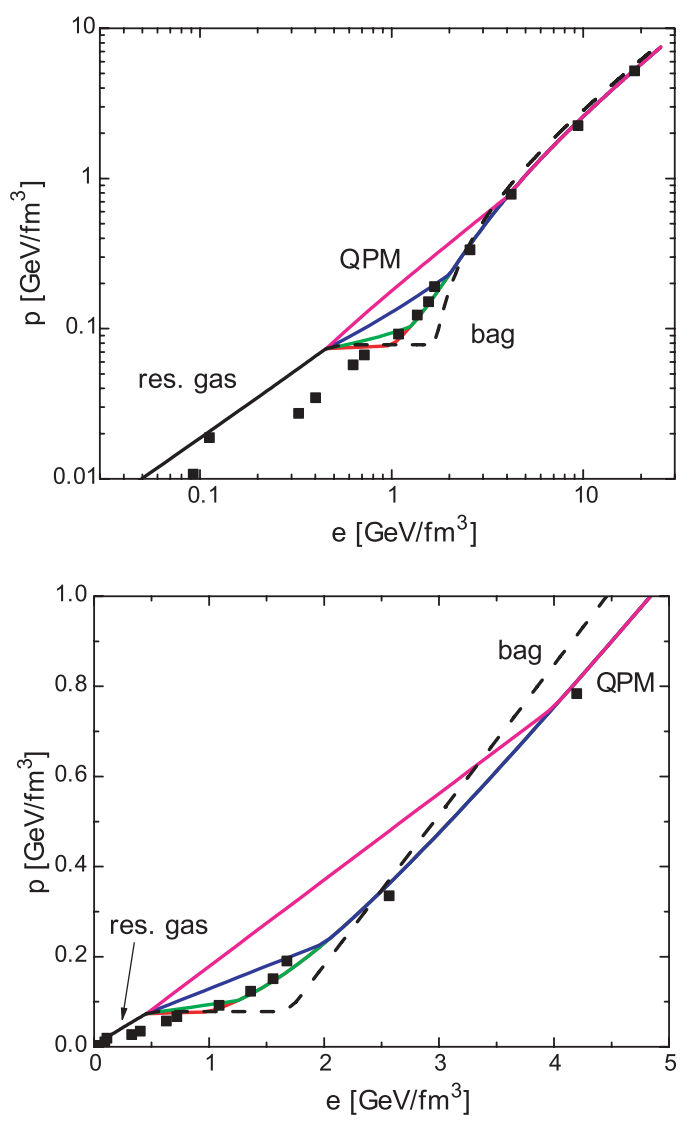

FIG. 15. (Color online) A family of equations of state for $N_{f}=2+1$, combining our QPM at high energy densities with a hadron resonance gas model ("res. gas") in the low-energy-density regime through linear interpolation. We show the range of energy densities relevant for collisions at RHIC. The solid lines show $p(e)$ for QPM(4.0), QPM(2.0), QPM(1.25), and QPM(1.0) (from top to bottom), where the numerical label indicates the matching point $e_{m}$ in $\mathrm{GeV} / \mathrm{fm}^{3}$. On the given scale, effects of varying $n_{B}$ between 0 and $0.5 \mathrm{fm}^{-3}$ are not visible. Lattice QCD data (squares) are from Ref. [63]. For comparison a bag model EoS ("bag") with a sharp first-order phase transition is also shown (dashed line). The bottom panel zooms in on the transition region, using a linear energy density scale.

The dashed line in Fig. 15 shows a Maxwell construction between the hadron resonance gas and a bag model EoS with $c_{s}^{2}=\frac{1}{3}$; this results in a strong first-order phase transition with latent heat $\Delta e_{\text {lat }}=1.1 \mathrm{GeV} / \mathrm{fm}^{3}$ ("EoS Q" in Refs. [1,68]).

Our construction differs from the approach explored in Ref. [2], where the hadron resonance gas is matched to an ideal quark-gluon gas with varying values for the latent heat $\Delta e_{\text {lat }}$. For example, by varying the latent heat in EoS Q from $\Delta e_{\text {lat }}=0.4$ to 0.8 and $1.6 \mathrm{GeV} / \mathrm{fm}^{3}$, the pressure $p\left(e_{0}, n_{B}=\right.$ 0 ) at a typical initial energy density $e_{0}=30 \mathrm{GeV} / \mathrm{fm}^{3}$ for central $\mathrm{Au}+\mathrm{Au}$ collisions at RHIC decreases by only $1.4 \%$ and $4.3 \%$, respectively, with correspondingly small changes in the entropy density $s_{0}$. In our approach, however, the entropy density $s_{0}$ at $e_{0}$ is given by lattice QCD and significantly $(\sim 15 \%)$ smaller. We note that our QPM(1.0) is similar to EOS $\mathrm{Q}$ in Refs. [1,68], except for the larger latent heat of EoS Q.

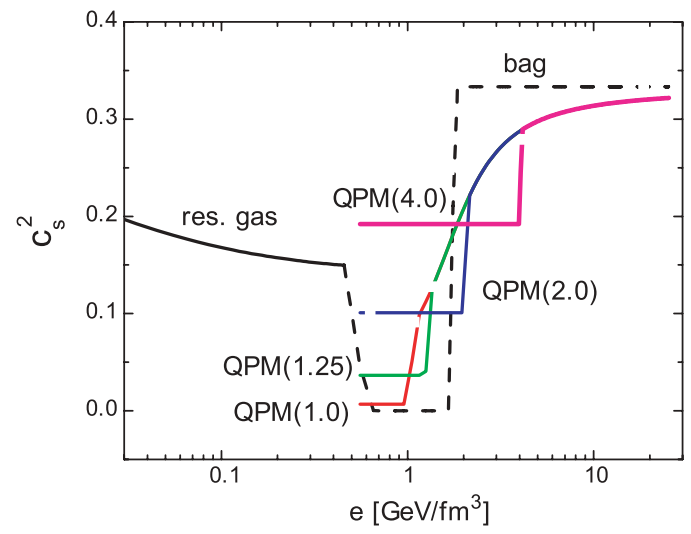

FIG. 16. (Color online) Squared speed of sound $c_{s}^{2}=\partial p / \partial e$ as a function of energy density $e$ along an isentropic expansion trajectory with $s / n_{B}=100$, for the EoS family QPM $\left(e_{m}\right)$ depicted in Fig. 15. Baryon density effects are not visible on the given scale as long as $n_{B}<0.5 \mathrm{fm}^{-3}$.

Figure 16 shows the corresponding squared speed of sound, $c_{s}^{2}$, as a function of energy density $e$. The linear interpolation between the hadron resonance gas at $e \leqslant e_{1}=0.45 \mathrm{GeV} / \mathrm{fm}^{3}$ and the QPM at $e \geqslant e_{m}$ leads to a region of constant sound speed for $e_{1} \leqslant e \leqslant e_{m}$. This constant increases monotonically with the matching point value $e_{m}$. For $e_{m}=3 \mathrm{GeV} / \mathrm{fm}^{3}$, the hadron resonance gas extrapolates smoothly to the QPM, with no "soft region" of small sound speed left over at all. In this case the typical phase transition signature of a softening of the EoS near $T_{c}$ is minimized, leading to minimal phase transition effects on the development of hydrodynamic flow.

\section{AZIMUTHAL ANISOTROPY AND TRANSVERSE MOMENTUM SPECTRA}

Equipped with our QCD-based family of equations of state, we can now explore the effects of fine structures in the EoS near $T_{c}$ on the evolution of hydrodynamic flow, by computing the transverse momentum spectra $d N /\left(d y p_{T} d p_{T} d \phi\right)$ and elliptic flow $v_{2}\left(p_{T}\right)$ for a variety of hadron species. To emphasize flow effects, we only consider directly emitted hadrons and neglect resonance decay distortions.

In noncentral heavy-ion collisions, the initial almondshaped cross section of the overlap zone perpendicular to the beam direction in coordinate space is converted into an azimuthally asymmetric momentum distribution by the appearance of a radially nonsymmetric flow governed by pressure gradients. If one assumes no transverse flow at a certain "initial time" $\tau_{0}$, at which the hydrodynamical expansion stage starts, the azimuthal asymmetry is determined by the acting pressure. Therefore, the azimuthal asymmetry is an ideal probe of the equation of state. In addition, the final anisotropy in the momentum distribution depends on the rescatterings among the particles and serves as measure of the degree of local thermalization.

The asymmetry is quantified by the harmonic coefficients of an expansion of the emitted hadrons transverse momentum spectra into a Fourier series in the azimuthal emission angle $\phi$ 
around the beam axis relative to the reaction plane (which is determined by the direction of the impact parameter $b$ ):

$$
\begin{aligned}
\frac{d N}{p_{T} d p_{T} d y d \phi}= & \frac{d N}{2 \pi p_{T} d p_{T} d y} \\
& \times\left[1+2 v_{2}\left(p_{T}, y\right) \cos 2 \phi+\cdots\right] .
\end{aligned}
$$

The second Fourier coefficient $v_{2}\left(p_{T}, y\right)=\langle\cos 2 \phi\rangle_{p_{T}, y}$ is called elliptic flow. We here exploit the $(2+1)$-dimensional relativistic hydrodynamic program package with Cooper-Frye freeze-out formalism, AZHYDRO, used in Refs. [1,4,5,8]. It assumes longitudinally boost-invariant expansion à la Bjorken. Clearly, this is appropriate only near midrapidity $y \approx 0$, but it is sufficient for purposes of our qualitative investigation here.

Different phenomenological equations of state of strongly interacting matter were proposed in previous studies [1-5,8$11,73]$, exhibiting a strong first-order phase transition with different values of latent heats $[1,2,4,8,11,73]$, a smooth but rapid crossover [5], or no phase transition at all [73]. These equations of state differ significantly in their high-density regions and softest points and in the speed of sound, which controls details of the developing flow pattern. Investigating the hydrodynamic consequences of different equations of state helps to establish benchmarks for tracing specific phase transition signatures and distinguishing them from other dynamical features (such as so far poorly explored viscous effects).

We emphasize, however, that we do not attempt here a systematic comparison with RHIC data. Previous studies $[1,2,5]$ have already qualitatively established that existing data are best described by an EoS with a phase transition or rapid crossover of significant strength (i.e., featuring a strong increase of the entropy and energy density within a narrow temperature interval) that exhibits both a soft part near $T_{c}$ and a hard part not too far above $T_{c}$. More quantitative statements about a preference of one form of the EoS over another require a discussion that goes beyond the pure ideal fluid dynamical approach discussed here, owing to well-known strong viscous effects on the evolution of elliptic flow in the late hadron resonance gas phase [76]. Studying the effects of EoS variations within a more complete framework that allows to account for nonideal fluid behavior in the very early and late stages of the fireball expansion is an important task for the future. Staying here within the ideal fluid approach, we do note, however, that our discussion improves over that presented in Ref. [5] by employing below $T_{c}$ a chemically nonequilibrated hadron resonance gas EoS that correctly reproduces the measured hadron yields, irrespective of the selected value for the hydrodynamic decoupling temperature.

\section{A. Top RHIC energy}

We employ P. Kolb's program package version 0.0 available from the OSCAR archive [68]. Although the study presented in Ref. [1] shows that at RHIC energies $(\sqrt{s} \sim 200 \mathrm{~A} \mathrm{GeV})$ most of the finally observed momentum anisotropy develops before the completion of the quark-hadron phase transition, the buildup of elliptic flow still occurs mostly in the temperature region where the lattice QCD data show significant deviations from an ideal quark-gluon gas. It is therefore of interest to investigate the effects of these deviations, and of variations of the exact shape of the EoS in the transition region, on the final elliptic flow in some detail, both at RHIC energies, where they are expected to matter, and at higher LHC energies where most (although not all [77]) of the anisotropic flow will develop before the system enters the phase transition region, thus reducing its sensitivity to the transition region.

We fix the initial conditions for top RHIC energy according to [1]

$$
s_{0}=110 \mathrm{fm}^{-3}, \quad n_{0}=0.4 \mathrm{fm}^{-3}, \quad \tau_{0}=0.6 \mathrm{fm} / c ;
$$

these parameters describing the initial conditions in the fireball center for central $(b=0) \mathrm{Au}+\mathrm{Au}$ collisions are required input for the AZHYDRO code [68]. From these initial conditions for central collisions the initial profiles for noncentral collisions are calculated by using the Glauber model [1]. For our EoS these values translate (independently of the QPM version used) into $e_{0}=29.8 \mathrm{GeV} / \mathrm{fm}^{3}, p_{0}=9.4 \mathrm{GeV} / \mathrm{fm}^{3}$, and $T_{0}=357 \mathrm{MeV}$. [Strictly speaking, because in the QPM the physical scale is set by $T_{c}$, varying $T_{c}$ in the range $170 \pm 10 \mathrm{MeV}$ would result in a variation of $e_{0}$ from 25 to $33 \mathrm{GeV} / \mathrm{fm}^{3}$ when keeping $s_{0}$ fixed (such as to maintain the same final charged particle multiplicity $\left.d N_{\mathrm{ch}} / d y \propto s_{0} \tau_{0}\right)$. We fix $T_{c}=170 \mathrm{MeV}$.]

Our calculations assume zero initial transverse velocity $\left(v_{T, 0}=0\right.$ at $\left.\tau=\tau_{0}\right)$. In the hadron phase, the Rapp-Kolb chemical off-equilibrium EoS [11] is used to account for frozen-out chemical reactions. The freeze-out criterion is $e_{\text {f.o. }}=0.075 \mathrm{GeV} / \mathrm{fm}^{3}$, corresponding to a freeze-out temperature of about $100 \mathrm{MeV}$. All hadrons are assumed to freeze-out at the same energy density.

The usual approach when analyzing data is to adjust the set of initial and final conditions to keep the transverse momentum spectra of a given set of hadron species fixed, and to then study the variation of $v_{2}$. Here we instead illustrate the impact of the EoS by using a fixed set of initial and freeze-out parameters. We explore $\mathrm{Au}+\mathrm{Au}$ collisions at a fixed impact parameter $b=5.2 \mathrm{fm}$, adjusted to best reproduce minimum bias data from the STAR Collaboration.

In Fig. 17 we show the transverse momentum spectra and differential elliptic flow for directly emitted $\Lambda, \Xi$, and $\Omega$ hyperons. These hadron species do not receive large resonance decay contributions, so by comparing the results for directly emitted particles with the measured spectra one can obtain a reasonable feeling for the level of quality of the model description. We show only results obtained with the two extreme equations of state, QPM(4.0) and the bag model EoS (see Fig. 15). The results for QPM(1.0) are very similar to those from the bag model EoS, although the latter features a larger latent heat. The two remaining equations of state [QPM(1.25) and QPM(2.0)] interpolate smoothly between the extreme cases shown in Fig. 17.

The upper panel in Fig. 17 shows that QPM(4.0) generates significantly larger radial flow, resulting in flatter $p_{T}$ spectra especially for the heavy hadrons shown here. This can be understood from Fig. 15 since this EoS does not feature a soft region with small speed of sound around $T_{c}$. Flatter $p_{T}$ spectra generically result in smaller Fourier coefficients $v_{2}\left(p_{T}\right)[1]$, 

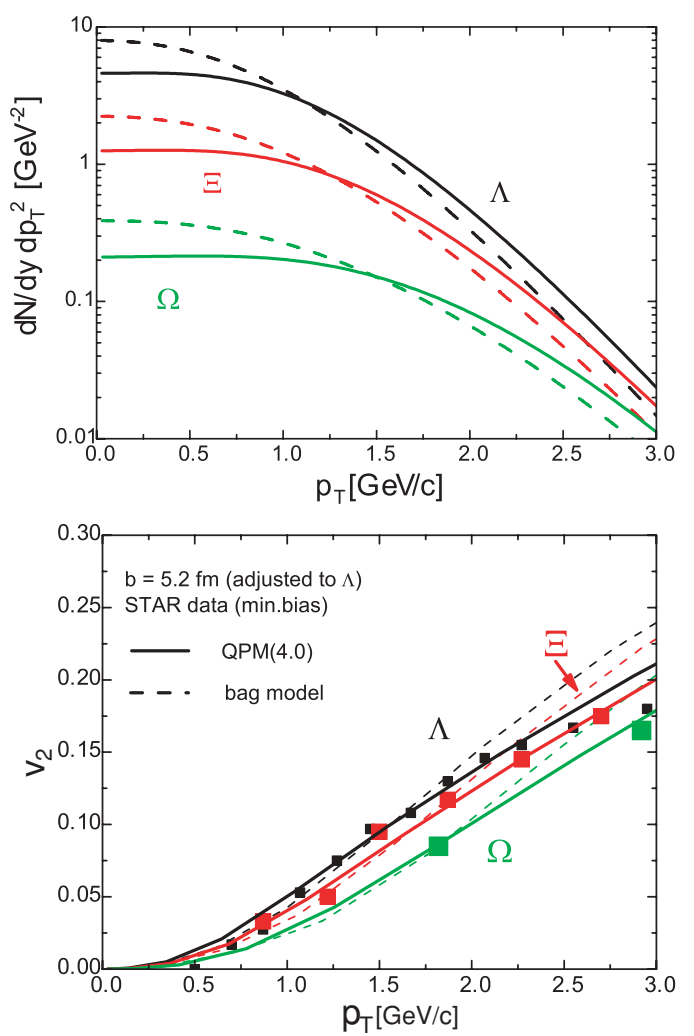

FIG. 17. (Color online) Transverse momentum spectra (top) and elliptic flow coefficient (bottom) for directly emitted strange baryons. The symbols represent STAR data [78] (see text for details). Solid and dashed curves are for EoS QPM(4.0) and the bag model EoS, respectively.

but the lower panel in Fig. 17 shows that, for $p_{T}<1.5 \mathrm{GeV} / c$, QPM(4.0) actually produces larger $v_{2}\left(p_{T}\right)$ than the bag model EoS. This implies that QPM(4.0) also produces a larger overall momentum anisotropy (i.e., $p_{T}$-integrated elliptic flow) than the bag model EoS, again owing to the absence of a soft region near $T_{c}$. Only at large $p_{T}>2 \mathrm{GeV} / c$, where the ideal fluid dynamic picture is known to begin to break down [6], does QPM(4.0) give smaller elliptic flow than the bag model EoS, as naively expected [1] from the flatter slope of the single-particle $p_{T}$ distribution.

The larger $v_{2}\left(p_{T}\right)$ at low $p_{T}<1.5 \mathrm{GeV} / c$ from QPM(4.0) is not favored by the data. In this sense we confirm the qualitative conclusion from earlier studies $[1,2,5]$ that the data are best described by an EoS with a soft region near $T_{c}$, followed by a rapid increase of the speed of sound $c_{s}$ above $T_{c}$.

\section{B. LHC estimates}

Predictions for $\mathrm{Pb}+\mathrm{Pb}$ collisions at the $\mathrm{LHC}$ involve a certain amount of guesswork about the initial conditions at the higher collision energy. Here we do not embark upon a systematic exploration of varying initial conditions, as proposed, for example, in Refs. [79], but simply guess conservatively

$$
s_{0}=330 \mathrm{fm}^{-3}, \quad \tau_{0}=0.6 \mathrm{fm} / c,
$$
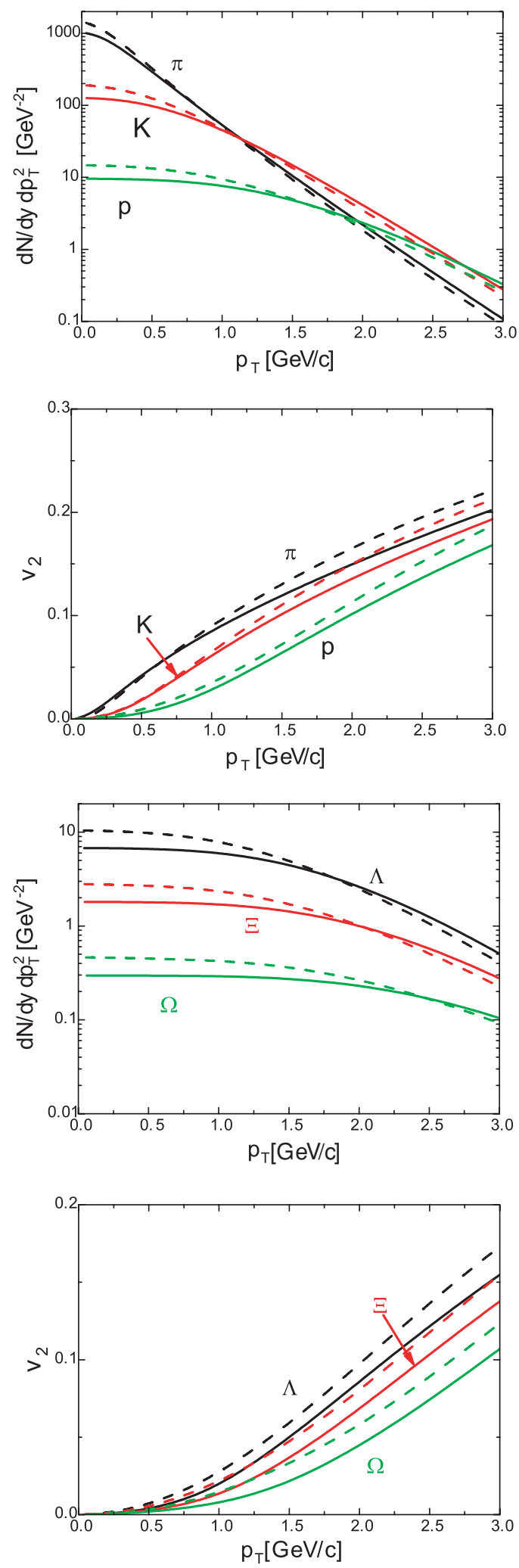

FIG. 18. (Color online) Transverse momentum spectra (top and third panels) and azimuthal anisotropy (second and bottom panels) for pions, kaons, and protons (top two panels) and strange baryons (bottom two panels). Initial conditions are according to Eq. (22). The spectra show only directly emitted hadrons. Solid and dashed curves are for EoS QPM(4.0) and the bag model EoS being similar to $\mathrm{QPM}(1.0)$, respectively. 
keeping all other parameters unchanged. This corresponds to final multiplicities that are three times those measured at RHIC. Within the QPM these initial parameters translate into $e_{0}=127 \mathrm{GeV} / \mathrm{fm}^{3}, p_{0}=42 \mathrm{GeV} / \mathrm{fm}^{3}$, and $T_{0}=515 \mathrm{MeV}$ for the peak values in central $\mathrm{Pb}+\mathrm{Pb}$ collisions. We again study collisions at impact parameter $b=5.2 \mathrm{fm}$, using the Glauber model to calculate the corresponding initial density profiles from these parameters.

Again we show results only for the two extreme equations of state, QPM(4.0) and the bag model EoS. Generally, the $p_{T}$ spectra for LHC initial conditions are flatter than for RHIC initial conditions, since the higher initial temperature and correspondingly longer fireball lifetime results in stronger radial flow. Figure 18 shows that again QPM(4.0), which lacks a soft region near $T_{c}$, generates even larger radial flow (i.e., a flatter $p_{T}$ spectra) than the bag model EoS [whose results are similar to those obtained with QPM(1.0)]. The radial flow effects are particularly strong for the heavy hyperons.

The overall momentum anisotropy (i.e., the $p_{T}$-integrated elliptic flow) does not increase very much between RHIC and LHC [1]. Since the LHC spectra are flatter (i.e., have more weight at larger $p_{T}$ than the RHIC spectra), the elliptic flow at fixed $p_{T}$ must therefore decrease. This is clearly seen when one compares the bottom panels of Figs. 17 and 18. The decrease is particularly strong for the hyperons at low $p_{T}$, where the LHC transverse momentum spectra become extremely flat.

\section{SUMMARY}

We have shown that available lattice QCD calculations give converging and robust results for the $\operatorname{EoS} p\left(e, n_{B}\right)$ in the region of large energy density. Baryon density effects were shown to be negligibly small for $n_{B}<0.5 \mathrm{fm}^{-3}$; that is, the EoS relevant for heavy-ion collisions at top RHIC and LHC energies is the same. In the transition region (i.e., for temperatures around $T_{c}$ ) different lattice calculations still exhibit quantitative differences, as seen particularly clearly in the bottom panel of Fig. 14. The lattice calculations examined here do not yet join smoothly at low energy densities (i.e., at $T<T_{c}$ ) to the hadron resonance gas model EoS with physical mass spectrum. Although our quasiparticle model covers all considered lattice QCD equations of state and serves as a reliable tool to connect thermodynamic quantities in a thermodynamically consistent way, it is not obvious that a reliable chiral extrapolation is feasible by simply replacing the quark mass parameters employed on the lattice by their physical values. If we do so we find significant quark mass effects only for energy densities below and close to $1 \mathrm{GeV} / \mathrm{fm}^{3}$ (i.e., below and in the vicinity of the hadronization phase transition).

In the present paper we therefore assumed as a working hypothesis the validity of the hadron resonance gas model EoS below $T_{c}$ (i.e., below an energy density of $e_{1}=0.45 \mathrm{GeV} / \mathrm{fm}^{3}$ ) and interpolated this EoS linearly to the robust high-energydensity branch from the chirally extrapolated QPM fit to the lattice QCD data. In doing so we arrive at a family of equations of state whose members $\mathrm{QPM}\left(e_{m}\right)$ are labeled by the matching point energy density $e_{m}$ where we join the QPM EoS. The resulting equations of state $\mathrm{QPM}\left(e_{m}\right)$ are available in the usual tabulated form on the OSCAR Web site [68]. We find that the uncertain intermediate region, which is bridged over by this interpolation procedure, has a small but non-negligible impact on the evolution of radial and elliptic flow in highenergy heavy-ion collisions, which is visible in the transverse momentum spectra and elliptic flow coefficients of various (directly emitted) hadron species. Existing RHIC data seem to favor those members of our family of equations of state that exhibit a soft region near $T_{c}$ followed by a rapid rise of the speed of sound toward the ideal gas value above $T_{c}$. We caution, however, that we did not perform a systematic study including simultaneous variations of the EoS and initial and final conditions and that event-by-event fluctuations [44-47] or viscous effects [80] may wash out differences among different sets of equations of state. More quantitative conclusions about the EoS require systematic investigations that match the ideal fluid description to viscous dynamical models for the very early and late stages of the fireball expansion; this is left for the future.

\section{ACKNOWLEDGMENTS}

We thank S. Fodor, S. Hands, P. Huovinen, F. Karsch, E. Laermann, A. Peshier, K. Redlich, and S. Wheaton for fruitful discussions. This work was supported by BMBF 06DR121/06DR136, GSI-FE, and Helmholtz VI, as well as by the U.S. Department of Energy under Contract No. DE-FG02-01ER41190.
[1] P. F. Kolb, J. Sollfrank, and U. Heinz, Phys. Lett. B459, 667 (1999); Phys. Rev. C 62, 054909 (2000); P. F. Kolb, P. Huovinen, U. Heinz, and H. Heiselberg, Phys. Lett. B500, 232 (2001); P. Huovinen, P. F. Kolb, U. Heinz, P. V. Ruuskanen, and S. A. Voloshin, ibid. B503, 58 (2001); P. F. Kolb and U. Heinz, in Quark Gluon Plasma 3, edited by R. C. Hwa and X. N. Wang (World Scientific, Singapore, 2004), p. 634.

[2] D. Teaney, J. Lauret, and E. V. Shuryak, Phys. Rev. Lett. 86, 4783 (2001); arXiv:nucl-th/0110037.

[3] E. V. Shuryak, Prog. Part. Nucl. Phys. 53, 273 (2004).

[4] P. Huovinen, in Quark Gluon Plasma 3, edited by R. C. Hwa and X. N. Wang (World Scientific, Singapore, 2004), p. 600;
P. Huovinen and P. V. Ruuskanen, Annu. Rev. Nucl. Part. Sci. 56, 163 (2006).

[5] P. Huovinen, Nucl. Phys. A761, 296 (2005).

[6] U. Heinz, J. Phys. G: Nucl. Part. Phys. 31, S717 (2005).

[7] The First Three Years of Operation of RHIC, Nucl. Phys. A757, 1 (2005).

[8] T. Hirano, Phys. Rev. C 65, 011901(R) (2001); T. Hirano and K. Tsuda, ibid. 66, 054905 (2002).

[9] D. Teaney, arXiV:nucl-th/0204023.

[10] R. Rapp, Phys. Rev. C 66, 017901 (2002).

[11] P. F. Kolb and R. Rapp, Phys. Rev. C 67, 044903 (2003).

[12] U. Heinz and P. F. Kolb, Nucl. Phys. A702, 269 (2002). 
[13] U. Heinz, Nucl. Phys. A721, 30 (2003).

[14] M. Gyulassy, in Structure and Dynamics of Elementary Matter, edited by W. Greiner et al., Nato Science Series II, Vol. 166 (Kluwer Academic, Dordrecht, 2004), p. 159.

[15] M. Gyulassy and L. McLerran, Nucl. Phys. A750, 30 (2005).

[16] E. V. Shuryak, Nucl. Phys. A750, 64 (2005).

[17] F. Karsch, E. Laermann, and A. Peikert, Phys. Lett. B478, 447 (2000).

[18] F. Karsch, Lect. Notes Phys. 583, 209 (2002).

[19] F. Karsch and E. Laermann, in Quark Gluon Plasma 3, edited by R. C. Hwa and X. N. Wang (World Scientific, Singapore, 2004), p. 1.

[20] A. Peshier, B. Kämpfer, O. P. Pavlenko, and G. Soff, Phys. Lett. B337, 235 (1994); Phys. Rev. D 54, 2399 (1996);A. Peshier, B. Kämpfer, and G. Soff, Phys. Rev. C 61, 045203 (2000); Phys. Rev. D 66, 094003 (2002).

[21] P. Levai and U. Heinz, Phys. Rev. C 57, 1879 (1998).

[22] R. A. Schneider and W. Weise, Phys. Rev. C 64, 055201 (2001).

[23] A. Rebhan and P. Romatschke, Phys. Rev. D 68, 025022 (2003); P. Romatschke, hep-ph/0210331.

[24] D. H. Rischke, Prog. Part. Nucl. Phys. 52, 197 (2004).

[25] J. Letessier and J. Rafelski, Phys. Rev. C 67, 031902(R) (2003).

[26] M. A. Thaler, R. A. Schneider, and W. Weise, Phys. Rev. C 69, 035210 (2004).

[27] Yu. B. Ivanov, V. V. Skokov, and V. D. Toneev, Phys. Rev. D 71, 014005 (2005); A. S. Khvorostukin, V. V. Skokov, V. D. Toneev, and K. Redlich, Eur. Phys. J. C48, 531 (2006).

[28] V. M. Bannur, Eur. Phys. J. C50, 629 (2007); Phys. Rev. E 73, 067401 (2006); hep-ph/0604158; Phys. Lett. B647, 271 (2007); Phys. Rev. C 75, 044905 (2007).

[29] A. Peshier and W. Cassing, Phys. Rev. Lett. 94, 172301 (2005).

[30] M. I. Gorenstein and S. N. Yang, Phys. Rev. D 52, 5206 (1995).

[31] M. Le Bellac, Thermal Field Theory (Cambridge University Press, Cambridge, 1996).

[32] J. I. Kapusta, Finite-Temperature Field Theory (Cambridge University Press, Cambridge, 1989).

[33] R. D. Pisarski, Nucl. Phys. A498, 423c (1989).

[34] M. Bluhm, diploma thesis, Technische Universität Dresden, August 2004 (unpublished); M. Bluhm, B. Kämpfer, R. Schulze, and D. Seipt, Eur. Phys. J. C49, 205 (2007).

[35] M. Bluhm, B. Kämpfer, and G. Soff, J. Phys. G 31, S1151 (2005); Phys. Lett. B620, 131 (2005).

[36] J. P. Blaizot, E. Iancu, and A. Rebhan, Phys. Rev. Lett. 83, 2906 (1999); Phys. Lett. B470, 181 (1999); Phys. Rev. D 63, 065003 (2001); Phys. Lett. B523, 143 (2001); Phys. Rev. D 68, 025011 (2003); in Quark Gluon Plasma 3, edited by R. C. Hwa and X. N. Wang (World Scientific, Singapore, 2004), p. 60.

[37] K. K. Szabo and A. I. Toth, JHEP 06 (2003) 008.

[38] M. Bluhm, B. Kämpfer, and G. Soff, Contribution to XLII Winter Meeting on Nuclear Physics, Bormio, Italy, Jan. 25-31, 2004 [hep-ph/0402252].

[39] S. Ejiri, F. Karsch, E. Laermann, and C. Schmidt, Phys. Rev. D 73, 054506 (2006).

[40] F. Karsch, Nucl. Phys. Proc. Suppl. 83, 14 (2000).

[41] C. R. Allton, S. Ejiri, S. J. Hands, O. Kaczmarek, F. Karsch, E. Laermann, and C. Schmidt, Phys. Rev. D 68, 014507 (2003); C. R. Allton, M. Döring, S. Ejiri, S. J. Hands, O. Kaczmarek, F. Karsch, E. Laermann, and K. Redlich, ibid. 71, 054508 (2005).
[42] These parameters differ significantly from the QPM parameters that optimally reproduce $c_{0}(T)$ from Ref. [17] (cf. Fig. 1). This is most likely a consequence of different algorithms and lattice QCD parameters used in Refs. [17] and [41]. It implies that the dependence of these lattice data on the quark masses and lattice spacing, combined with the presently incompletely controlled [66] continuum extrapolation, is too complex to be fully captured by our QPM. If we compute the pressure $p\left(T, \mu_{B}=0\right)$ [i.e. $\left.c_{0}(T)\right]$ from the QPM with the parameters used in Figs. 2 and 3 (which were tuned to $c_{2}(T)$ from Ref. [41]), adjusting the extra parameter $B\left(T_{c}\right)$, which is not needed for the $c_{n}(T), n \geqslant 2$, to the value $B\left(T_{c}\right)=0.61 T_{c}^{4}$, we find a result that is about $10 \%$ larger than that shown in Fig. 1 for large $T$ and rises somewhat more rapidly in the vicinity of $T_{c}$, resulting in a larger entropy density.

[43] M. Bluhm, B. Kämpfer, R. Schulze, and D. Seipt, Acta Phys. Hung. A 27, 397 (2006).

[44] M. Asakawa, U. Heinz, and B. Müller, Phys. Rev. Lett. 85, 2072 (2000); Nucl. Phys. A698, 519 (2002).

[45] S.-Y. Jeon and V. Koch, Phys. Rev. Lett. 85, 2076 (2000).

[46] O. Socolowski, F. Grassi, Y. Hama, and T. Kodama, AIP Conf. Proc. 739, 649 (2005); Phys. Rev. Lett. 93, 182301 (2004).

[47] C. E. Aguiar, Y. Hama, T. Kodama, and T. Osada, Nucl. Phys. A698, 639 (2002).

[48] R. V. Gavai and S. Gupta, Phys. Rev. D 71, 114014 (2005); 72, 054006 (2005); 73, 014004 (2006).

[49] R. V. Gavai and S. Gupta, Phys. Rev. D 67, 034501 (2003); (private communication, March 2006).

[50] L. D. Landau and E. M. Lifshitz, Fluid Mechanics, 2nd Ed. (Butterworth and Heinemann, 1987).

[51] F. Karsch (private communication, January 2006).

[52] J. Cleymans, B. Kämpfer, M. Kaneta, S. Wheaton, and N. Xu, Phys. Rev. C 71, 054901 (2005).

[53] F. Becattini, J. Manninen, and M. Gazdzicki, Phys. Rev. C 73, 044905 (2006)

[54] J. Cleymans, H. Oeschler, K. Redlich, and S. Wheaton, Phys. Rev. C 73, 034905 (2006), and references therein.

[55] S. Wheaton (private communication, July 2005).

[56] M. A. Halasz, A. D. Jackson, R. E. Shrock, M. A. Stephanov, and J. J. M. Verbaarschot, Phys. Rev. D 58, 096007 (1998).

[57] K. Rajagopal and F. Wilczek, in At the Frontier of Particle Physics, Vol. 3, edited by M. Shifman (World Scientific, Singapore, 2001) p. 2061.

[58] M. A. Stephanov, Prog. Theor. Phys. Suppl. 153, 139 (2004), and references therein; Int. J. Mod. Phys. A 20, 4387 (2005).

[59] Z. Fodor and S. D. Katz, JHEP 03 (2002) 014; 04 (2004), 050.

[60] Ph. de Forcrand and O. Philipsen, Nucl. Phys. B673, 170 (2003).

[61] C. Schmidt, C. R. Allton, S. Ejiri, S. J. Hands, O. Kaczmarek, F. Karsch, and E. Laermann, "The quark mass and mu dependence of the QCD chiral critical point," Nucl. Phys. Proc. Suppl. 119, 517 (2003) [arXiV:hep-lat/0209009].

[62] A. Peikert, Ph.D. thesis, Universität Bielefeld, 2000.

[63] F. Karsch, K. Redlich, and A. Tawfik, Eur. Phys. J. C 29, 549 (2003); Phys. Lett. B571, 67 (2003).

[64] Z. Fodor, S. D. Katz, and K. K. Szabo, Phys. Lett. B568, 73 (2003).

[65] F. Karsch, E. Laermann, and A. Peikert, Nucl. Phys. B605, 579 (2001).

[66] Y. Aoki, Z. Fodor, S. D. Katz, and K. K. Szabo, Phys. Lett. B643, 46 (2006); Y. Aoki, G. Endrodi, Z. Fodor, S. D. Katz, and K. K. Szabo, Nature (London) 443, 675 (2006). 
[67] M. Cheng et al., Phys. Rev. D 74, 054507 (2006).

[68] See the link to the hydrodynamic code AZHYDRO at http://nt3.phys.columbia.edu/people/molnard/OSCAR/ and references given there. A tabulated version of the EoS for a hadron resonance gas in chemical equilibrium (EOS Q files named aa ${ }^{*}$.dat) is available at http://www.physics.ohiostate.edu/ froderma/.

[69] F. Karsch, AIP Conf. Proc. 842, 20 (2006)].

[70] C. Bernard et al., Phys. Rev. D 55, 6861 (1997); PoS LAT2005, 156 (2006).

[71] Y. Aoki, Z. Fodor, S. D. Katz, and K. K. Szabo, JHEP 01 (2006) 089.

[72] R. Hagedorn, Nuovo Cimento Suppl. 3, 147 (1965).

[73] J. Sollfrank et al., Phys. Rev. C 55, 392 (1997).
[74] R. Dashen, S. Ma, and H. J. Bernstein, Phys. Rev. 187, 345 (1969).

[75] P. Braun-Munzinger, D. Magestro, K. Redlich, and J. Stachel, Phys. Lett. B518, 41 (2001).

[76] T. Hirano, U. Heinz, D. Kharzeev, R. Lacey, and Y. Nara, Phys. Lett. B636, 299 (2006).

[77] T. Hirano, U. Heinz, D. Kharzeev, R. Lacey, and Y. Nara, J. Phys. G: Nucl. Part. Phys. 34, S879 (2007).

[78] J. Adams et al. (STAR Collaboration), Phys. Rev. Lett. 95, 122301 (2005).

[79] R. J. Fries, J. I. Kapusta, and Y. Li, nucl-th/0604054; A. Krasnitz and R. Venugopalan, Phys. Rev. Lett. 84, 4309 (2000).

[80] D. Teaney, Nucl. Phys. A715, 817 (2003). 